\title{
ARTICLE
}

\section{Calcium channel blockers reduce severe fever with thrombocytopenia syndrome virus (SFTSV) related fatality}

Hao Li ${ }^{1}$, Lei-Ke Zhang ${ }^{2,3,4}$, Shu-Fen Li ${ }^{2}$, Shao-Fei Zhang ${ }^{1}$, Wei-Wei Wan ${ }^{2}$, Yu-Lan Zhang ${ }^{2}$, Qi-Lin Xin ${ }^{2}$, Ke Dai $^{1}$, Yuan-Yuan Hu ${ }^{1}$, Zhi-Bo Wang ${ }^{1}$, Xiang-Tao Zhu ${ }^{2,3}$, Yu-Jie Fang ${ }^{2,3}$, Ning Cui ${ }^{5}$, Pan-He Zhang ${ }^{1}$, Chun Yuan ${ }^{5}$, Qing-Bin Lu ${ }^{6}$, Jie-Ying Bai ${ }^{7}$, Fei Deng $\mathbb{1 D}^{2,3,4}$, Geng-Fu Xiao ${ }^{2,3,4}$, Wei Liu ${ }^{1}$ and Ke Peng iD $^{2,3,4}$

Severe fever with thrombocytopenia syndrome (SFTS), an emerging tick-borne infectious disease caused by a novel phlebovirus (SFTS virus, SFTSV), was listed among the top 10 priority infectious diseases by the World Health Organization due to its high fatality of $12 \%-50 \%$ and possibility of pandemic transmission. Currently, effective anti-SFTSV intervention remains unavailable. Here, by screening a library of FDA-approved drugs, we found that benidipine hydrochloride, a calcium channel blocker (CCB), inhibited SFTSV replication in vitro. Benidipine hydrochloride was revealed to inhibit virus infection through impairing virus internalization and genome replication. Further experiments showed that a broad panel of CCBs, including nifedipine, inhibited SFTSV infection. The anti-SFTSV effect of these two CCBs was further analyzed in a humanized mouse model in which CCB treatment resulted in reduced viral load and decreased fatality rate. Importantly, by performing a retrospective clinical investigation on a large cohort of 2087 SFTS patients, we revealed that nifedipine administration enhanced virus clearance, improved clinical recovery, and remarkably reduced the case fatality rate by $>5$-fold. These findings are highly valuable for developing potential host-oriented therapeutics for SFTS and other lethal acute viral infections known to be inhibited by CCBs in vitro.

Cell Research (2019) 29:739-753; https://doi.org/10.1038/s41422-019-0214-z

\section{INTRODUCTION}

Tick-borne infectious diseases are recognized as a growing threat to public health at a global level. ${ }^{1}$ Severe fever with thrombocytopenia syndrome (SFTS) is an emerging tick-borne infectious disease caused by a novel phlebovirus (SFTS virus, SFTSV) in the family Phenuiviridae of the order Bunyavirales. ${ }^{2}$ The disease causes a wide clinical spectrum, ranging from mild febrile disease accompanied by thrombocytopenia and/or leukocytopenia, to hemorrhagic fever, clinical encephalitis, multiple organ failure, and eventually death. ${ }^{2,3}$ SFTS was first identified in China and subsequently reported in South Korea and Japan with a fatality rate of $12 \%-50 \%{ }^{2,4-6}$ Due to its high fatality and the possibility of causing pandemic transmission, SFTS was listed among the top 10 priority infectious disease with urgent need for research by the World Health Organization.

Tick-to-human transmission is the primary route by which people are infected with SFTSV, and Haemaphysalis longicornis tick is the main reservoir and vector of SFTSV. ${ }^{8,9}$ Haemaphysalis longicornis is native to East Asia and has established populations in the Australasian and Western Pacific Regions. ${ }^{10-13}$ Migratory birds are natural hosts of $\mathrm{H}$. longicornis ticks and it was proposed that birds carrying SFTSV-infected ticks could contribute to the longdistance spread of the virus through migratory flyways. ${ }^{14,15}$ Very recently, this tick species has been reported to spread in the United States, first observed to infest the sheep in New Jersey and then identified in seven states and the suburb of New York City. ${ }^{16,17}$ The presence of $H$. longicornis ticks brings the possibility of pandemic transmission of SFTSV in North America.

Despite the high case fatality, vaccines or specific antivirals for SFTSV are currently not available. Ribavirin, which was suggested as a potential antiviral therapeutic drug for SFTS, was reported to be inefficient in improving the disease outcome in several retrospective studies.,18 Our recent study revealed that the ribavirin therapy reduced the case fatality rate (CFR) in patients with a viral load below $10^{6}$ copies $/ \mathrm{mL}$ (tested at hospital admission), but showed no effect on the patients who had a viral load over $10^{6}$ copies/mL. ${ }^{6}$ Favipiravir and 2'-Fluoro-2'-deoxycytidine $\left(2^{\prime}\right.$-FdC) were reported to have anti-SFTSV efficacy; ${ }^{19,20}$ however, these compounds have not yet been approved for clinical use and the drug safety should be carefully evaluated particularly for 2'-FdC, which has been reported to be incorporated into the liver DNA in animal experiments. ${ }^{21}$ In two recent studies amodiaquine and hexachlorophene were identified to inhibit SFTSV infection, but evaluation was only performed in cultured cell models and the in vivo drug efficacy still awaits further investigation in animal models. ${ }^{22,23}$ As such, there is an

\footnotetext{
${ }^{1}$ State Key Laboratory of Pathogen and Biosecurity, Beijing Key Laboratory of Vector Borne and Natural Focus Infectious Diseases, Beijing Institute of Microbiology and Epidemiology, Beijing 100071, China; ${ }^{2}$ State Key Laboratory of Virology, Wuhan Institute of Virology, Chinese Academy of Sciences, Wuhan, Hubei 430071 , China; ${ }^{3}$ University of Chinese Academy of Sciences, Beijing 100049, China; ${ }^{4}$ Wuhan National Biosafety Laboratory, Mega-Science Center for Bio-Safety Research, Chinese Academy of Sciences, Wuhan, Hubei 430071, China; ${ }^{5}$ The 154 Hospital, People's Liberation Army, Xinyang, Henan, China; ${ }^{6}$ School of Public Health, Peking University, Beijing, China and ${ }^{7}$ Laboratory Animal Center, Academy of Military Medical Sciences, Beijing, China
}

Correspondence: Wei Liu (liuwei@bmi.ac.cn) or Ke Peng (pengke@wh.iov.cn)

These authors contributed equally: Hao Li, Lei-Ke Zhang.

Received: 11 May 2019 Accepted: 23 July 2019

Published online: 23 August 2019 
740

urgent need for developing new anti-SFTSV therapeutics with potent efficacy, especially for patients suffering from severe infection and having high viral loads with a high possibility of progressing to fatal outcome. Screening U.S. Food and Drug Administration (FDA) approved drug library is an effective strategy to identify potential antivirals and has been successfully applied against emerging viruses such as Zika virus ${ }^{24}$ and Ebola virus. ${ }^{25}$ Given the approved status of these drugs, the identified drug candidates have the potential to be rapidly advanced to clinical settings as therapeutic countermeasures. ${ }^{25}$

In search of anti-SFTSV compound, we screened a FDAapproved drug library and found that benidipine hydrochloride and nifedipine, two calcium channel blockers (CCBs), inhibited SFTSV replication in vitro. Benidipine hydrochloride was revealed to inhibit SFTSV infection through impairing viral internalization and genome replication. The anti-SFTSV efficacy of these two CCBs was further demonstrated in $\mathrm{C} 57 \mathrm{BL} / 6$ and humanized mouse models. A retrospective clinical investigation on a large cohort of 2087 SFTS patients showed that nifedipine can distinctly inhibit SFTSV infection and reduce SFTS-associated CFR. This study provided the first clinical evidence indicating that CCB treatment may be developed as an effective countermeasure to treat SFTSV infection and, potentially, to treat other lethal viral infections that are known to be inhibited by CCBs in vitro.

\section{RESULTS}

A screen of FDA-approved drugs identified compounds with antiSFTSV activity

Screening FDA-approved drugs for anti-viral compounds is an effective strategy for repurposed drug application as the clinical safety of identified drug candidate has been extensively evaluated. For this reason, we screened a library of 700 FDAapproved drugs for anti-SFTSV activity (Fig. 1a). Vero cell monolayers were treated with each drug or vehicle (DMSO, dissolvent of the drugs) with mycophenolic acid (MPA) as the positive control for $1 \mathrm{~h}$, and then inoculated with SFTSV at a multiplicity of infection (MOI) of 1 . At 36 hours post infection (hpi), cells were fixed, and the infected cells were detected by immunofluorescence analysis (IFA) using antibody against the viral nucleoprotein (NP) protein. The percentage of SFTSV-infected cells upon each drug treatment was calculated by automated imaging and quantitative analysis. The $Z$ factor was calculated as previously described ${ }^{26}$ and was determined to be 0.61 indicating that the primary screening was robust. The results from two experimental runs were compared, yielding a correlation coefficient of 0.80 , which confirmed the reproducibility of the results (Fig. 1b). Fourteen drugs significantly inhibited virus infection (inhibition rate $>70 \%$ ) without causing cytotoxicity (survive rate $>70 \%$ ) at the concentration of $10 \mu \mathrm{M}$ (Fig. 1b; Supplementary information, Table S1). To validate the anti-SFTSV activity of these 14 drugs, Vero cells were treated with each drug at the concentrations of $0.1,1,5,10,20 \mu \mathrm{M}$, respectively, and then infected with SFTSV $(\mathrm{MOI}=1)$. At $36 \mathrm{hpi}$, inhibition rates were analyzed as described above. Seven drugs displayed anti-SFTSV activity in a dose-dependent manner (all $R^{2}>0.800$, Fig. 1c).

Benidipine hydrochloride inhibits SFTSV infection in different cell lines

Of the seven drugs identified in the primary screening, benidipine hydrochloride displayed the strongest inhibitory effect of SFTSV infection $(>90 \%)$ at the concentration of $10 \mu \mathrm{M}$. We next determined the half cytotoxic concentration $\left(\mathrm{CC}_{50}\right)$ and half maximal inhibitory concentration $\left(\mathrm{IC}_{50}\right)$ of benidipine hydrochloride on SFTSV infection. Briefly, Vero cells were treated with benidipine hydrochloride $1 \mathrm{~h}$ prior to SFTSV infection and then harvested at $36 \mathrm{hpi}$, and relative intracellular vRNA level was measured with quantitative real-time PCR (qRT-PCR). The $C C_{50}$ and
$\mathrm{IC}_{50}$ of benidipine hydrochloride were determined to be $96.92 \mu \mathrm{M}$ and $1.412 \mu \mathrm{M}$, respectively, resulting in a selection index of 68.6 in Vero cells (Fig. 1d, e). Immunological focus assay further revealed that benidipine hydrochloride exhibited a dose-dependent inhibition effect on the production of SFTSV progeny virions (Fig. 1f). The inhibitory effect of benidipine hydrochloride on SFTSV infection was also evaluated in Huh7 cells, a permissive cell line to SFTSV infection, and a dose-dependent anti-SFTSV effect was similarly observed (Fig. 1g, h).

Benidipine hydrochloride inhibits SFTSV infection by both interfering with virus internalization and reducing viral genome replication

We then investigated the inhibitory mechanism of benidipine hydrochloride against SFTSV infection (Supplementary information, Fig. S1). First, the virucidal effect of benidipine hydrochloride on SFTSV virion was examined. SFTSV was incubated with the indicated concentrations of benidipine hydrochloride for $1 \mathrm{~h}$ or treated with heat, and then the treated viruses were added to Vero cells. We found that intracellular levels of SFTSV vRNA were comparable between mock-treated and benidipine hydrochloridetreated groups, whereas heat-treated SFTSV group showed background level of vRNA at 24 hpi (Supplementary information, Fig. S2a), suggesting that benidipine hydrochloride treatment has no virucidal effect on SFTSV.

Then we explored whether benidipine hydrochloride affects SFTSV infection at the stage of entry. Benidipine hydrochloride was added to Vero cells at $1 \mathrm{~h}$ prior to SFTSV infection, and removed at $1 \mathrm{hpi}$. We found that the intracellular levels of SFTSV vRNA were significantly lower in benidipine hydrochloride-treated groups (all $P<0.05$, Fig. 2a), suggesting that benidipine hydrochloride inhibits SFTSV infection during entry, a process that includes binding, internalization into endosomes, and fusion of virus envelope with endosomal membranes. ${ }^{27}$

To test the effect of benidipine hydrochloride on viral binding, Vero cells were treated with benidipine hydrochloride or vehicle, and then incubated with SFTSV at $4{ }^{\circ} \mathrm{C}$ for $1 \mathrm{~h}$ to synchronize viral binding. Then unbound SFTSV virions were washed away with phosphate-buffered saline (PBS), and the relative levels of bound SFTSV virions were measured. As shown in Supplementary information, Fig. S2b, no significant difference of SFTSV vRNA was observed between benidipine hydrochloride-treated and vehicle (DMSO)-treated samples, suggesting that benidipine hydrochloride does not affect SFTSV binding.

Next, benidipine hydrochloride was evaluated for its potential effect on viral internalization. It was recently reported that SFTSV enters cells through the endocytosis pathway; ${ }^{28,29}$ thus blocking virus fusion, such as addition of ammonium chloride, would allow investigation of pre-fusion events including binding and internalization. Vero cells were treated with benidipine hydrochloride or vehicle (DMSO), infected with SFTSV in the presence of ammonium chloride to prevent membrane fusion. Afterwards, relative intracellular levels of virions were measured. The observed levels of internalized virions were significantly lower in benidipine hydrochloride-treated cells (all $P<0.05$, Fig. 2b). Virus internalization was further investigated with microscopy analysis. Vero cells were treated with benidipine hydrochloride or vehicle (DMSO), respectively, followed by infection with SFTSV in the presence of ammonium chloride for $1 \mathrm{~h}$. Afterwards, intracellular viral particles were stained with NP polyclonal antibody and cross sections of the middle of the cells were imaged. Infection was performed with a high $\mathrm{MOI}$ of 20 to increase the chance of viral particles being captured by the cross section. Intracellular viral particles were then quantified for both benidipine hydrochloride- and DMSO-treated cells. As shown in Fig. 2c, d, benidipine hydrochloride treatment significantly reduced the number of intracellular viral particles compared to the DMSO control. Since previous results showed that benidipine hydrochloride treatment does not affect virus 
a

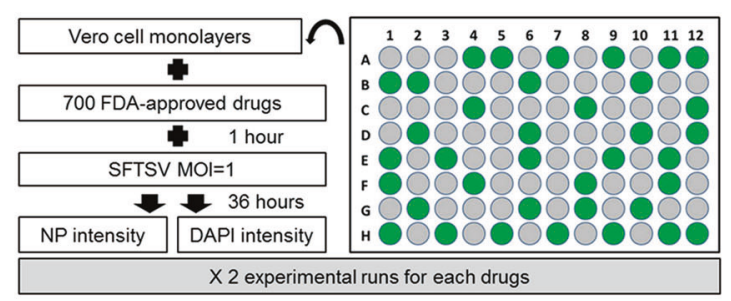

C
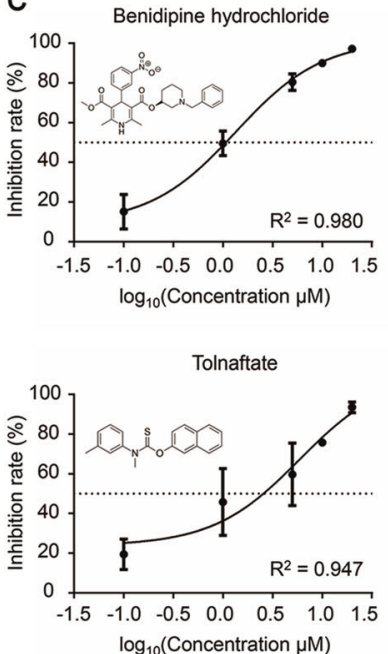

$\log _{10}($ Concentration $\mu \mathrm{M})$

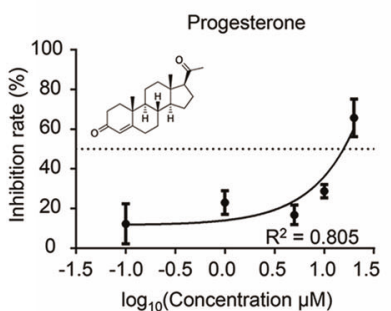

b
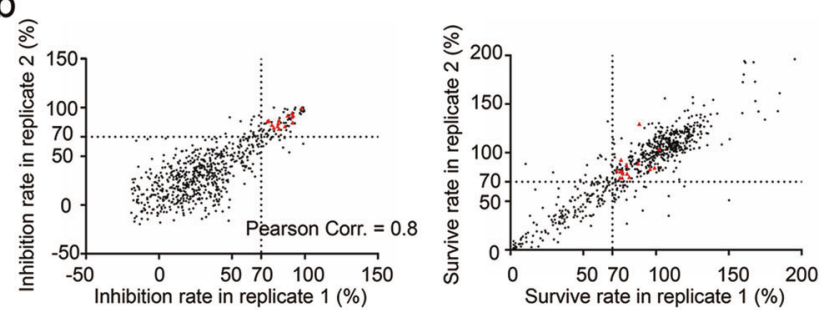

f
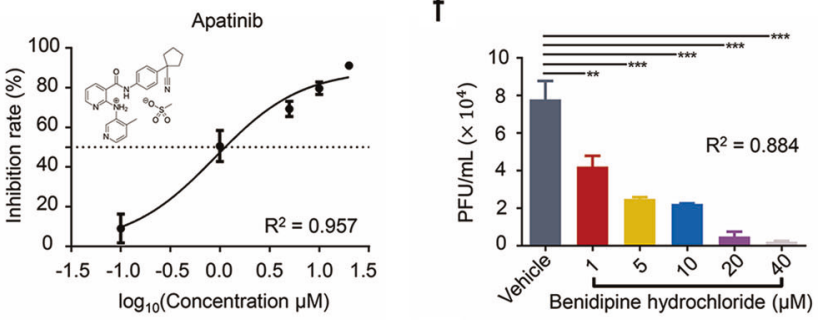

g
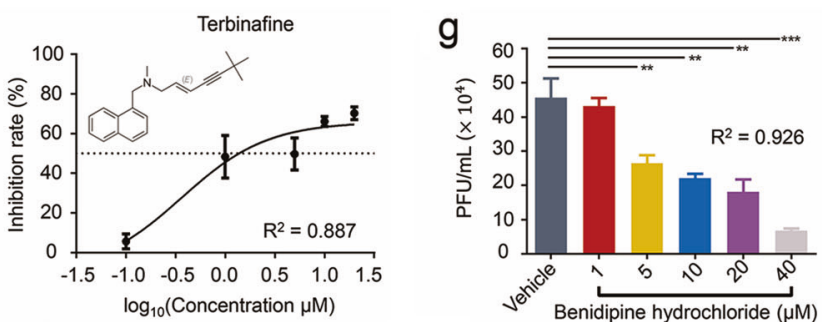

e

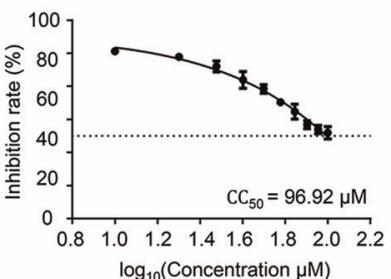

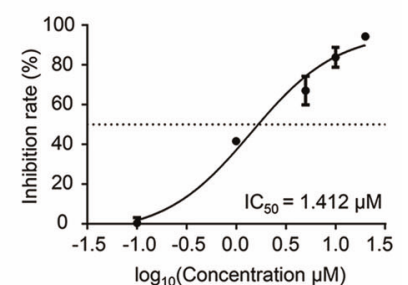

h

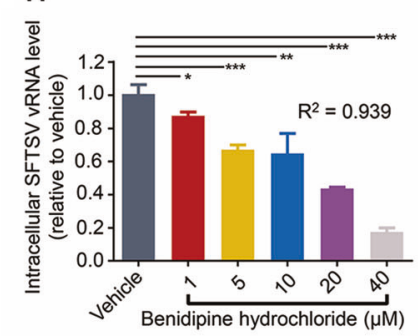

Fig. 1 Cell-based high-throughput screening of FDA-approved drug library identified benidipine hydrochloride as an inhibitor of SFTSV replication. a Cell-based high-throughput screening to identify inhibitors of SFTSV replication. b Analysis of screen reproducibility. Dots represent the inhibition rate (left) and cell survive rate (right) for each drug from two replicates of the screen. Red dots indicated 14 drugs that inhibited virus infection (inhibition rate $>70 \%$ ) without causing significant cytotoxicity (survive rate $>70 \%$ ). c Dose-dependent inhibitory effects of the seven selected compounds analyzed in Vero cells. Vero cell monolayers were treated with each drug at the indicated concentrations for $1 \mathrm{~h}$, and then inoculated with SFTSV at MOI of 1 . At $36 \mathrm{hpi}$, cells were fixed, and the infected cells were detected through immunofluorescence analysis using antibody against NP protein. The chemical structure of each compound is displayed. d Survival rate analysis of benidipine hydrochloride treatment in Vero cells. $\mathrm{CC}_{50}: 50 \%$ cytotoxic concentration. e Dose-dependent inhibition effects of benidipine hydrochloride on SFTSV infection. $\mathrm{IC}_{50}: 50 \%$ inhibitory concentration. $\mathrm{f}$ Inhibition effect of benidipine hydrochloride on the production of infectious SFTSV progeny virions in Vero cells. Vero cells were treated with serial concentrations of benidipine hydrochloride and infected with SFTSV, and virus titer in the supernatant at $16 \mathrm{hpi}$ was determined. $\mathbf{g}$ Inhibition effect of benidipine hydrochloride on the production of infectious SFTSV progeny virions in Huh7 cells. Experiment in Huh7 cells was performed as described in $\mathbf{f}$. $\mathbf{h}$ Inhibition effect of benidipine hydrochloride on intracellular SFTSV vRNA level in Huh7 cells. Huh7 cells were treated with serial concentrations of benidipine hydrochloride and infected with SFTSV. At $48 \mathrm{hpi}$, intracellular RNA was extracted and the vRNA level of SFTSV was measured with qRT-PCR. Comparison of mean values between benidipine hydrochloride-treated group and vehicle (DMSO) group (f-h) was analyzed by Student's $t$ test. ${ }^{*} P<0.05 ;{ }^{* *} P<0.01 ;{ }^{* *} P<0.001 . R^{2}(\mathbf{c}, \mathbf{f}-\mathbf{h})$ was estimated by nonlinear regression model (curve fit)

binding, these results suggest that the drug inhibits SFTSV internalization.

Following internalization, viral envelope fuses with cellular membrane to release viral ribonucleoprotein. The potential effect of benidipine hydrochloride on SFTSV fusion was then evaluated by detecting virus-induced syncytium formation as described before. $^{28}$ Significant syncytium formation of SFTSV-infected cells was observed after cells were incubated with citrate-phosphate buffer $(0.1 \mathrm{M}$ citric acid, $0.2 \mathrm{M}$ sodium dihydrogen orthophosphate, $\mathrm{pH}$ 5.0), and no significant difference of syncytium formation was observed between benidipine hydrochloridetreated and vehicle (DMSO)-treated cells (Supplementary information, Fig. S2c), suggesting that benidipine hydrochloride does not affect viral fusion.

We further tested whether benidipine hydrochloride inhibits SFTSV replication in the post entry phase. Vero cells were infected with SFTSV in the presence of ammonium chloride to synchronize virus infection at the stage of fusion, and at $3 \mathrm{hpi}$, ammonium chloride was removed and benidipine hydrochloride was added. We found that SFTSV replication level was significantly lower in 
a

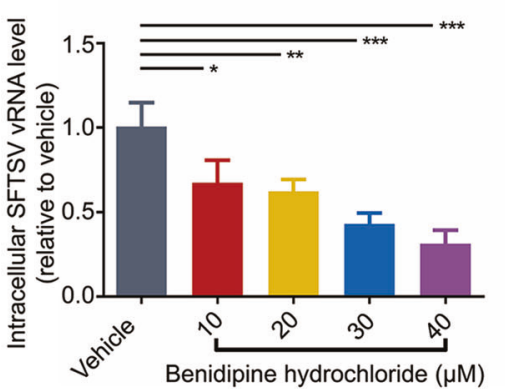

C

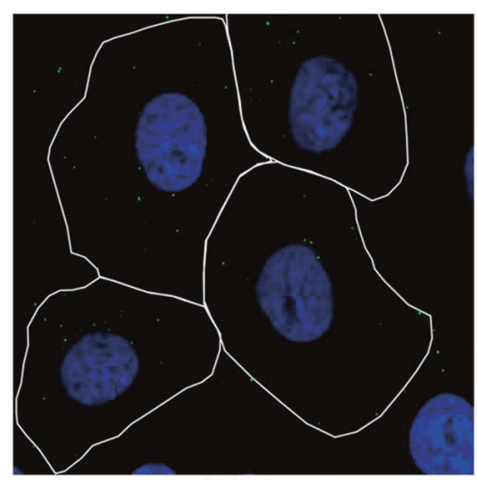

Vehicle

f

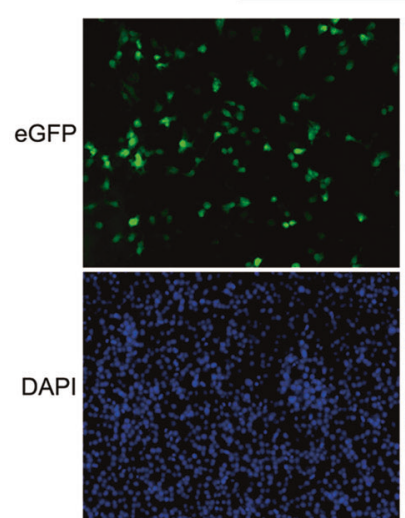

Vehicle b

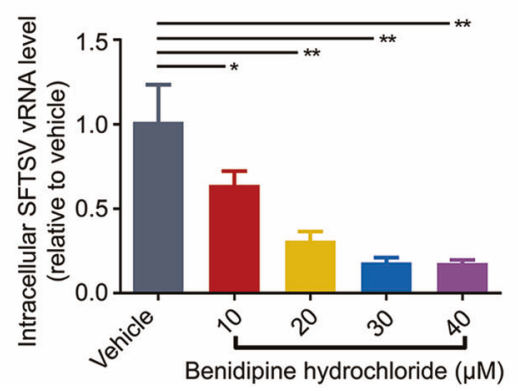

Benidipine hydrochloride $(\mu \mathrm{M})$

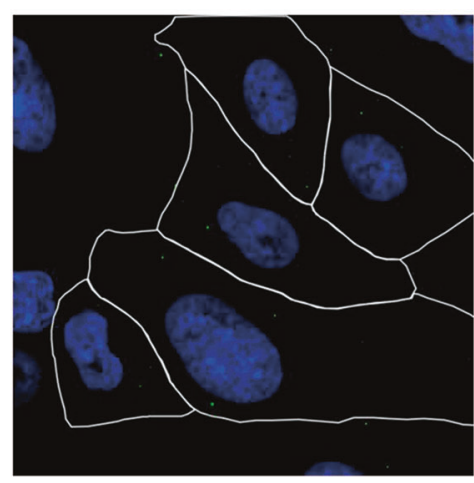

Benidipine hydrochloride
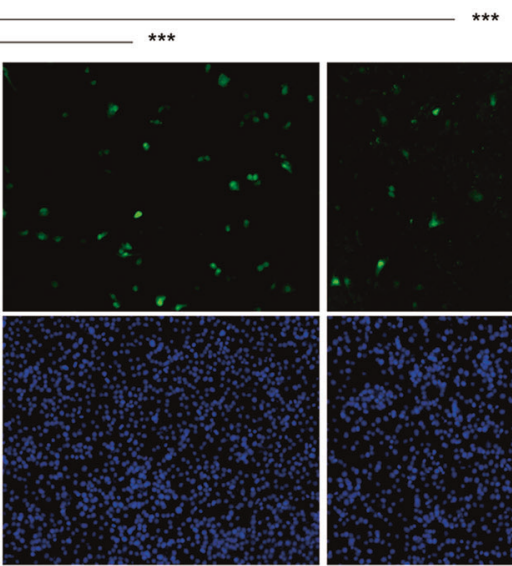

10

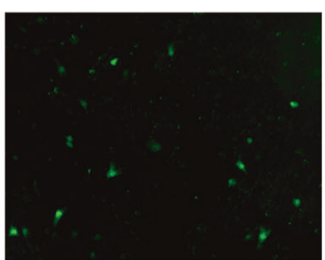

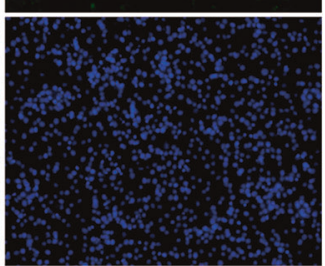

20

Benidipine hydrochloride $(\mu \mathrm{M})$

e

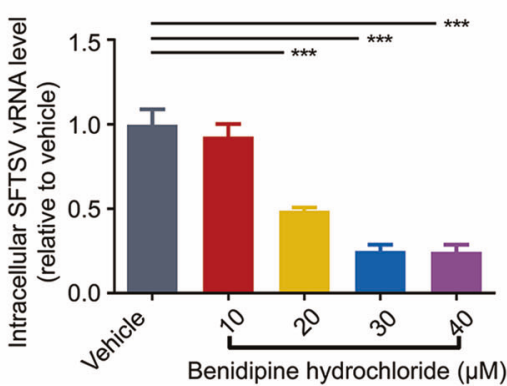

d

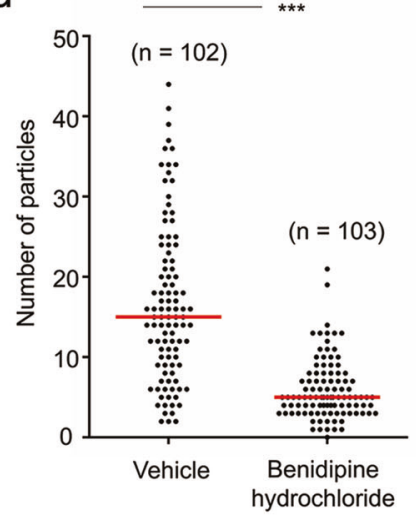

g
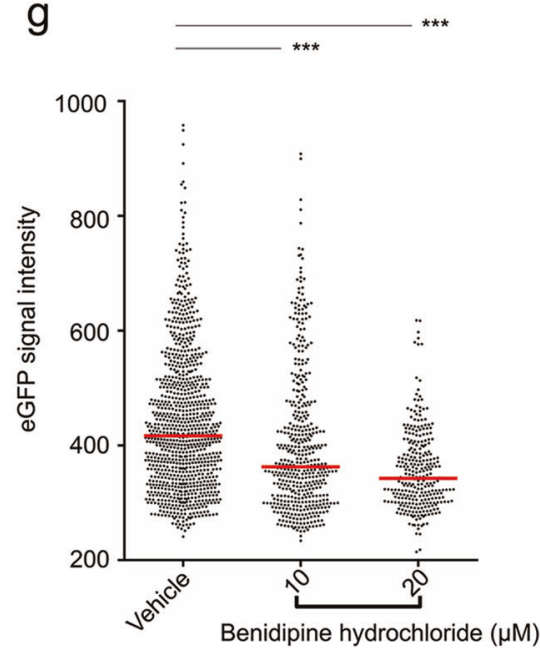

Fig. 2 In vitro anti-SFTSV mechanism of benidipine hydrochloride. a Effect of benidipine hydrochloride on SFTSV entry. Vero cells were treated with benidipine hydrochloride or Vehicle (DMSO) and then infected with SFTSV. At $1 \mathrm{hpi}$, benidipine hydrochloride was removed, and relative intracellular vRNA level of SFTSV was measured at $16 \mathrm{hpi}$. b Effect of benidipine hydrochloride on the internalization of SFTSV. Vero cells were treated with benidipine hydrochloride or vehicle (DMSO) and then infected with SFTSV in the presence of ammonium chloride. At $3 \mathrm{hpi}$, cell surface-bound virions were removed by tryspin, and relative vRNA level of internalized SFTSV was measured. c Fluorescence microscopy analysis of effect of benidipine hydrochloride on SFTSV internalization. Vero cells were infected with SFTSV at MOI of 20 in the presence of benidipine hydrochloride $(20 \mu \mathrm{M})$ or vehicle (DMSO) for $1 \mathrm{~h}$. Cells were washed with PBS, briefly treated with trypsin and then labeled with NP antibody, and cross sections of the middle of the cells were imaged. Representative images of drug- or vehicle (DMSO)-treated cells were shown. White lines indicate cell boundaries. d Quantification of intracellular viral particles of each cell in c was performed and analyzed. Red line indicates median value of each group. $\mathrm{n}$ values indicate number of cells analyzed. e Effect of benidipine hydrochloride on SFTSV postentry events. Vero cells were infected with SFTSV and then benidipine hydrochloride was added at 3 hpi. Relative intracellular vRNA level of SFTSV was measured at $16 \mathrm{hpi}$. $\mathbf{f}$, g Effect of benidipine hydrochloride on SFTSV MG activity. BSRT7 cells were transfected with SFTSV MG system and vehicle (DMSO) or benidipine hydrochloride was added at the indicated concentration. MG activity was assessed by the percentage of the eGFP-positive cells (f) and the eGFP signal intensity at single-cell level (g). Red lines indicate median values. Comparisons of mean values $(\mathbf{a}, \mathbf{b}$, and $\mathbf{e})$ and median values $(\mathbf{d}, \mathbf{g})$ between two groups were performed by Student's $t$-test and Mann-Whitney test, respectively. Comparison of the percentage of MG activity positive cells between two groups (f) was analyzed by $\chi^{2}$ test. ${ }^{*} P<0.05$; ${ }^{* *} P<0.01$; $* * * P<0.001$ 
benidipine hydrochloride-treated cells at $16 \mathrm{hpi}$ (all $P<0.001$ for concentrations $\geq 20 \mu \mathrm{M}$, Fig. 2e), suggesting that benidipine hydrochloride also inhibits SFTSV replication in the post entry phase.

To investigate whether benidipine hydrochloride inhibits viral RNA replication, a SFTSV mini-genome (MG) system was constructed (Supplementary information, Fig. S3a). Briefly, a plasmid containing the SFTSV M genome fragment was constructed and the coding sequence of viral glycoprotein was replaced with the eGFP sequence to express the reporter gene. This reporter plasmid and the plasmids expressing NP and $L$ proteins, were co-transfected into BSRT7 cells. Expression of NP and $L$ proteins will drive the synthesis of $M$ fragment, leading to eGFP expression, which can be quantified with microscopy analysis (referred to as MG activity). Indeed, while cotransfection of all three plasmids led to a significant eGFP production (Supplementary information, Fig. S3b, Vehicle), leaving out the L plasmid or applying MPA, a non-nucleoside inhibitor of eukaryotic IMP dehydrogenase, ${ }^{30}$ during transfection, resulted in only a background level of eGFP expression (Supplementary information, Fig. S3b), confirming the specificity of the MG system. The MG system was then used to examine whether benidipine hydrochloride can inhibit vRNA synthesis. BSRT7 cells transfected with the MG system were treated with benidipine hydrochloride and the eGFP expression was recorded and analyzed. Benidipine hydrochloride treatment significantly reduced the number of eGFP-positive cells in comparison with vehicle (DMSO) treatment (both $P<0.001$, Fig. 2f). Furthermore, the eGFP signals at singlecell level were analyzed and, as shown in Fig. $2 \mathrm{~g}$, benidipine hydrochloride treatment resulted in lower intracellular eGFP signal intensity in comparison with vehicle (DMSO) treatment (both $P<0.001)$. Taken together, these results suggest that benidipine hydrochloride inhibits SFTSV RNA synthesis.

To further analyze whether benidipine hydrochloride inhibits SFTSV budding, Vero cells were treated with benidipine hydrochloride and infected with SFTSV. At 16 hpi, SFTSV RNAs from the supernatant and infected cells were both extracted and quantified. We found that benidipine hydrochloride treatment caused no further reduction of released viral RNA besides inhibiting viral RNA replication (Supplementary information, Fig. S2d), indicating that benidipine hydrochloride does not affect SFTSV budding. Taken together, these results suggested that benidipine hydrochloride inhibits SFTSV infection through interfering with viral internalization and reducing viral genome replication (Supplementary information, Fig. S1).

\section{CCBs inhibit SFTSV infection through reducing cellular $\mathrm{Ca}^{2+}$} uptake

Since benidipine hydrochloride is a CCB that reduces intracellular $\mathrm{Ca}^{2+}$ level, ${ }^{31}$ we next aimed to determine whether the anti-viral effect is associated with reduced intracellular $\mathrm{Ca}^{2+}$ level. Vero cells were infected with SFTSV, either with $\mathrm{Ca}^{2+}$-free medium or normal medium. At 16 and 24 hpi, intracellular level of SFTSV vRNA was measured with qRT-PCR. As shown in Fig. 3a, the intracellular levels of SFTSV vRNA were significantly lower in cells cultured with $\mathrm{Ca}^{2+}$-free medium (both $P<0.001$ ). Similarly, treatment with BAPTA-AM, a calcium chelator, during SFTS infection, also reduced vRNA level in a dose-dependent manner $\left(R^{2}=0.934\right.$, Fig. 3b). We further analyzed whether SFTSV infection induced $\mathrm{Ca}^{2+}$ influx. The intracellular $\mathrm{Ca}^{2+}$ level during SFTSV infection was measured with an intracellular calcium-sensitive dye. As shown in Fig. 3c, SFTSV infection induced $\mathrm{Ca}^{2+}$ influx over time and treatment with benidipine hydrochloride inhibited $\mathrm{Ca}^{2+}$ influx. These results suggested that increased intracellular $\mathrm{Ca}^{2+}$ level is beneficial for SFTSV infection and benidipine hydrochloride inhibits SFTSV infection through reducing the intracellular $\mathrm{Ca}^{2+}$ level.

We then tested a panel of CCBs to investigate whether inhibition of SFTSV replication is a general feature of these drugs.
Briefly, Vero cells were incubated with drugs and then infected with SFTSV, and at $36 \mathrm{hpi}$, cells were fixed and the infection rate was quantified with IFA as described above. As shown in Fig. 3d, several CCBs, including benidipine hydrochloride, nicardipine, cilnidipine, nisoldipine, manidipine, felodipine, amlodipine besylate, amlodipine, nitrendipine, nimodipine, and nifedipine, inhibited NP signal by $>50 \%$, suggesting that inhibition of SFTSV replication is a general feature of these CCBs. Notably, nifedipine, one of the most widely used drugs for treating hypertension and atherosclerosis in China, ${ }^{32,33}$ inhibited SFTSV infection in a dosedependent manner without causing any cytotoxicity effect (Fig. 3e). The effect of nifedipine on SFTSV-induced $\mathrm{Ca}^{2+}$ influx was determined and, similar to benidipine hydrochloride, nifedipine also inhibited SFTSV-induced $\mathrm{Ca}^{2+}$ influx (Fig. 3c).

Since calcium channel is the target of these two drugs, we next analyzed whether disruption of calcium channel would lead to reduced SFTSV infection. Both benidipine hydrochloride and nifedipine inhibit L-type calcium channels, ${ }^{34,35}$ and $\mathrm{Ca}_{\mathrm{v}} 1.2$, a L-type calcium channel, is reported to be targeted by nifedipine. ${ }^{36}$ Therefore, the $\mathrm{Ca}_{\mathrm{v}} 1.2$ was chosen for further analysis. Huh7 cells were transfected with specific small interfering RNAs (siRNAs) against $\mathrm{Ca}_{\mathrm{v}} 1.2$ for $48 \mathrm{~h}$ and then infected with SFTSV for 24 or $48 \mathrm{~h}$, followed by analysis of intracellular viral NP level. Transfection with the three siRNAs resulted in reduced $\mathrm{Ca}_{\mathrm{v}} 1.2$ level (Fig. $3 \mathrm{f}$ ) without affecting the cell viability (Supplementary information, Fig. S4a). Depletion of $\mathrm{Ca}_{\mathrm{v}} 1.2$ significantly reduced the intracellular NP level (Fig. 3f; Supplementary information, Fig. S4b), indicating restricted SFTSV infection upon $\mathrm{Ca}_{\mathrm{v}} 1.2$ knockdown. Taken together, these results suggested that CCB treatment inhibited SFTSV infection through reducing virus-induced $\mathrm{Ca}^{2+}$ influx and the $\mathrm{Ca}_{\mathrm{v}} 1.2$ calcium channel might be an important target for the inhibition of SFTSV by these two CCBs.

Benidipine hydrochloride and nifedipine inhibit SFTSV infection in the C57BL/6 mouse model

We next evaluated the in vivo effect of CCBs on SFTSV infection in C57BL/6 mouse, a verified animal model for SFTSV infection. ${ }^{37}$ Benidipine hydrochloride and nifedipine were chosen for evaluation as benidipine hydrochloride has the strongest inhibitory effect on SFTSV infection in vitro and nifedipine represents the most widely used CCB in China. Briefly, 5-week-old female C57BL/ 6 mice were challenged intraperitoneally with SFTSV, and at $1 \mathrm{hpi}$, intragastrically treated with benidipine hydrochloride, nifedipine, or vehicle for seven days. The viral loads in spleen samples were significantly decreased in benidipine hydrochloride-treated mice at $1,3,5$, and 7 days post infection (dpi) $(P=0.008, P=0.012, P=$ 0.002 , and $P=0.008$, respectively, Fig. $4 \mathrm{a})$, and in nifedipinetreated mice at 3,5 , and $7 \mathrm{dpi}(P=0.024, P=0.016$, and $P=0.006$, respectively). A significant decrease of serum viral loads was also observed in benidipine hydrochloride-treated mice at 3 and 5 dpi $(P=0.028$ and $P=0.036$, respectively, Fig. 4b), and in nifedipinetreated mice at $3 \mathrm{dpi}(P=0.045)$. Consistently, less viral antigenpositive cells was present in the spleens of mice treated with benidipine hydrochloride or nifedipine as revealed by immunohistochemistry analysis (Supplementary information, Fig. S8).

Increase of megakaryocytes in the spleens of SFTSV-infected C57BL/6 mice has been reported and proposed to be related to the compensation for SFTSV-induced circulating platelet (PLT) depletion. ${ }^{37}$ Our hematoxylin and eosin $(\mathrm{H} \& \mathrm{E})$ staining results showed that less megakaryocytes were observed in the spleens of mice treated with benidipine hydrochloride or nifedipine at 3 and $5 \mathrm{dpi}$ (Supplementary information, Fig. S8). In addition, the mice treated with benidipine hydrochloride showed higher levels of PLT count ( $P=0.023$, Supplementary information, Fig. S9) and serum calcium $(P=0.038)$, and a lower level of aspartate aminotransferase (AST) $(P=0.031)$ at $5 \mathrm{dpi}$, in comparison with vehicle-treated mice. These results indicated that benidipine hydrochloride or 


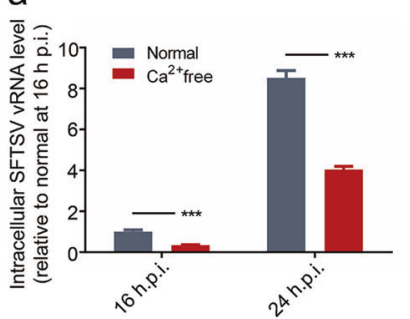

b

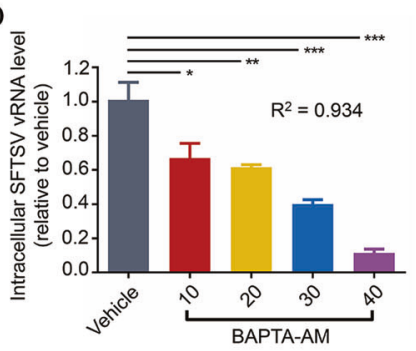

C

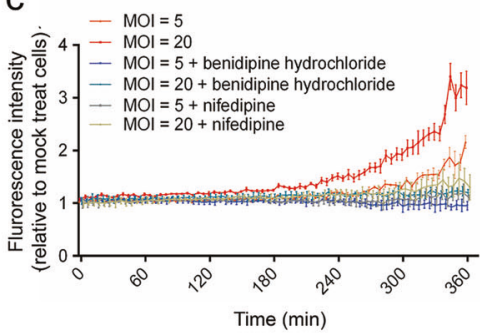

d

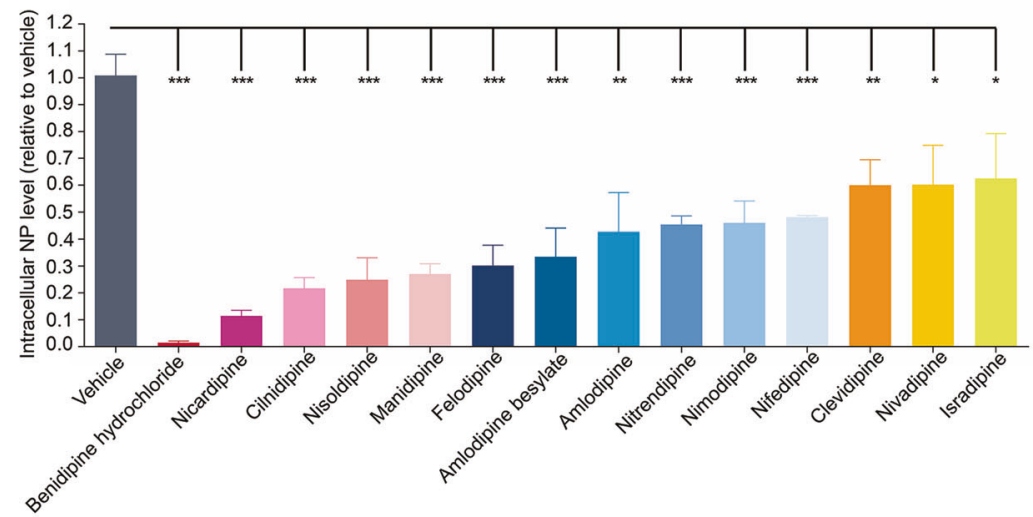

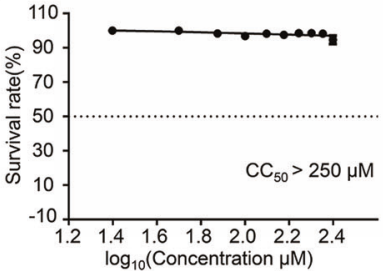

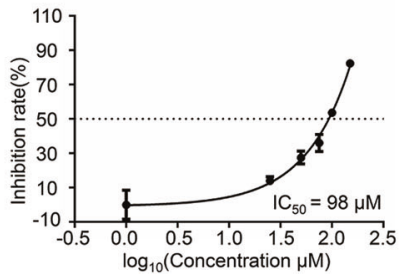

$f$

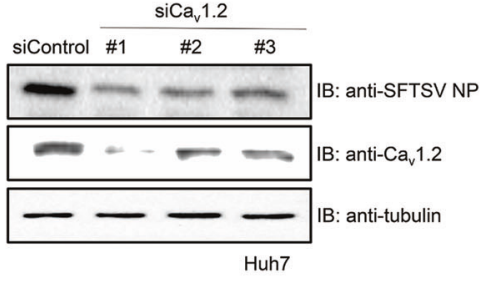

Fig. 3 In vitro anti-SFTSV activity of CCBs. a Vero cells were cultured in $\mathrm{Ca}^{2+}$-free or normal medium and then infected with SFTSV (MOI $=1$ ). At 16 or $24 \mathrm{hpi}$, relative intracellular SFTSV vRNA level was measured by qRT-PCR. b Relative intracellular vRNA level of SFTSV in Vero cells upon treatment with BAPTA-AM at $24 \mathrm{hpi}$. $\mathrm{c}$ Intracellular $\mathrm{Ca}^{2+}$ concentration in SFTSV-infected cells. Vero cells were infected with SFTSV at the indicated $\mathrm{MOI}$ in the presence of benidipine hydrochloride or nifedipine or vehicle (DMSO). Relative intracellular Ca ${ }^{2+}$ level was determined by fluorescence of Fluo-4NW. d Relative intracellular SFTSV NP level in the infected Vero cells. Vero cells were treated with the indicated 14 CCBs or vehicle (DMSO), infected with SFTSV at MOI of 1 and the relative NP level was determined by immunofluorescence with NP antibody at 36 hpi. e Survival rate analysis (left) and dose-dependent inhibition effects (right) of nifedipine treatment in Vero cells. $\mathbf{f}$ Relative intracellular SFTSV NP level in SFTSV-infected Huh7 cells. Cells were transfected with siRNA against L-type calcium channel Cav1.2 for $48 \mathrm{~h}$ and then infected with SFTSV for $48 \mathrm{~h}$, followed by western blot analysis of intracellular NP level. Comparison of mean values (a, b, and d) between two groups was analyzed by Student's $t$-test. ${ }^{*} P<0.05$; ${ }^{* *} P<0.01 ;{ }^{* * *} P<0.001$

nifedipine could reduce SFTSV loads and alleviate SFTSV-induced pathogenesis in C57BL/6 mouse model.

Benidipine hydrochloride and nifedipine treatment inhibited SFTSV infection and reduced fatality rate in the humanized mouse model

Although the above experiments clearly demonstrated the antiSFTSV effects of benidipine hydrochloride and nifedipine in the C57BL/6 mouse model, the correlation between reduced viral load and fatality rate could not be evaluated as C57BL/6 mouse is a non-lethal infection model for SFTSV. We therefore analyzed the possibility of employing humanized mouse model as a lethal SFTSV infection animal model. As shown in Supplementary information, Figs. S10, S11, SFTSV infection of humanized mice led to multiple organ infection, reduced PLT and white blood cell (WBC) counts and elevated alanine aminotransferase (ALT) and AST levels, which are similar to the clinical features of SFTSV patients. ${ }^{2,6}$ In addition, as shown in Fig. 4d, SFTSV infection resulted in lethal outcome in humanized mouse model. Taken together, these results suggest that the humanized mouse model can be used for evaluating the efficacy of anti-SFTSV drugs. The
anti-SFTSV efficacy of these two drugs was then tested in the humanized mouse model. The humanized mice were divided into vehicle, benidipine hydrochloride and nifedipine treatment groups, and virus infection and drug treatment were performed as described in Materials and Methods. As shown in Fig. 4c, both benidipine hydrochloride and nifedipine treatment significantly reduced the viral load compared to vehicle $(0.3 \%$ carboxymethyl cellulose solution) control at 5 and $7 \mathrm{dpi}$. Notably, virus titre was the highest during this period of infection (Fig. 4c).

More importantly, in the vehicle control group SFTSV infection led to the fatality rate of $57.1 \%(4 / 7)$ and nifedipine treatment significantly decreased the fatality rate to $0 \%(0 / 6, P=0.035)$ (Fig. 4d). A clear reduction of fatality rate was also observed in benidipine hydrochloride treatment group $(16.7 \%, 1 / 6)$ (Fig. $4 d)$, although the difference was not significant $(P=0.167)$ potentially due to the limited sample size. In the vehicle control group, death occurred on $5(n=1), 6(n=1)$ and $8(n=2)$ dpi. The single death event in the benidipine hydrochloride group occurred on $7 \mathrm{dpi}$. In both cases, these mice died in a period when the viral load was the highest. When the viral load ( $\log _{10}$-trasmitted) on $5 \mathrm{dpi}$ was analyzed, a distinct correlation between high viral load and death 
b

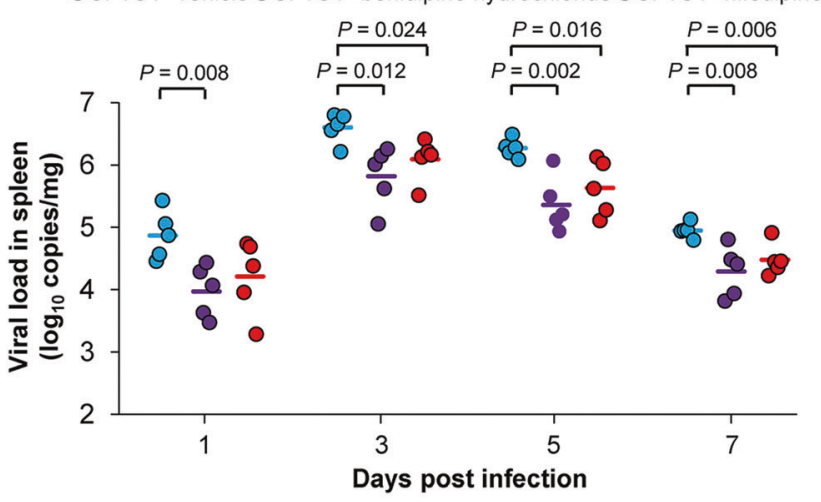

C

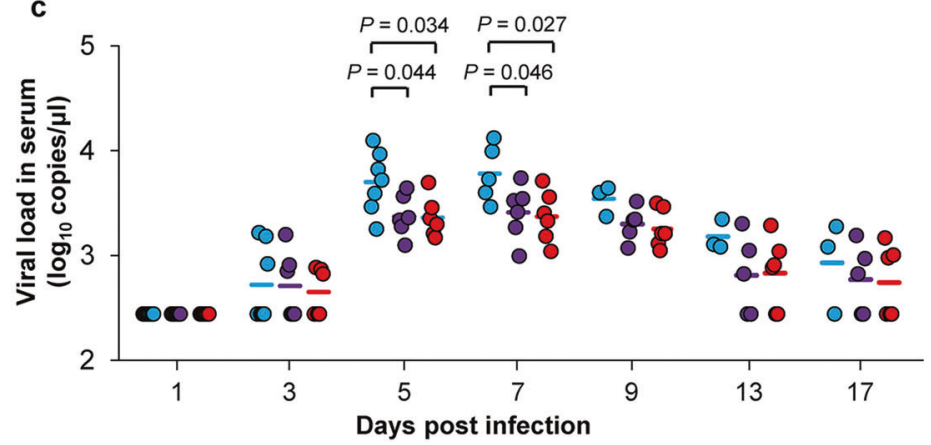

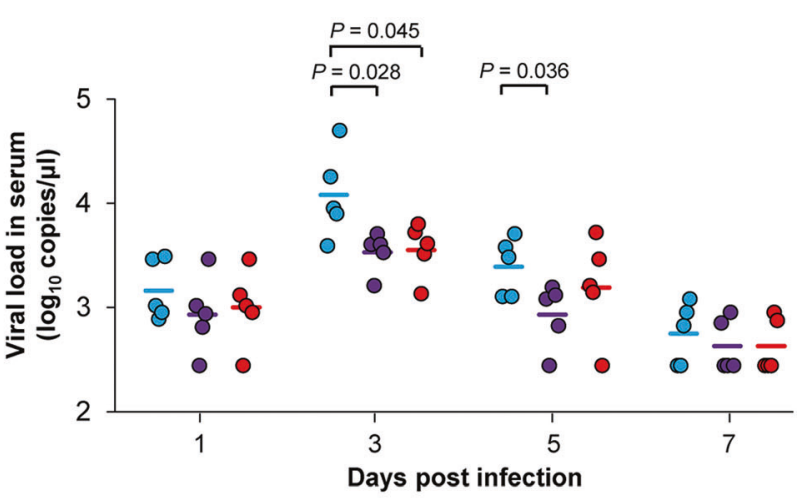

d

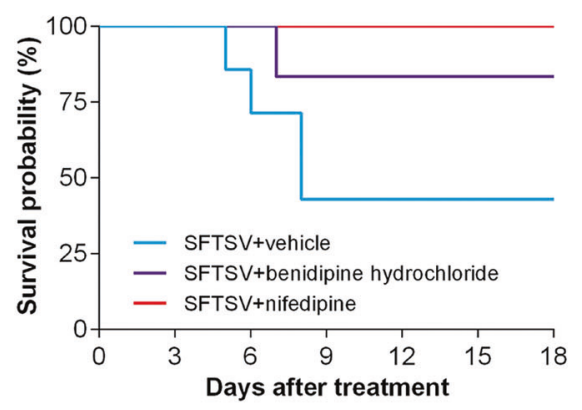

Fig. 4 Anti-SFTSV effect of benidipine hydrochloride and nifedipine in mouse models. a, b Spleen (a) or serum (b) viral loads in SFTSV-infected C57BL/6 mice (five for each group) treated with benidipine hydrochloride, nifedipine, or vehicle (0.3\% carboxymethyl cellulose solution), were measured by qRT-PCR at 1, 3, 5, and 7 dpi. c Serum viral loads in SFTSV-infected humanized mice treated with benidipine hydrochloride ( $\mathrm{n}=$ $6)$, nifedipine $(n=6)$, or vehicle $(0.3 \%$ carboxymethyl cellulose solution) $(n=7)$, were measured by qRT-PCR at $1,3,5,7,9,13$, and 17 dpi. $d$ Analysis of benidipine hydrochloride and nifedipine treatment on survival probability in humanized mice. The Kaplan-Meier method was used to analyze time-to-event data. The mean value of each group (a-c) was indicated by horizontal line. The significance of the difference between mean values was determined by Student's $t$-test. $P$ values $<0.05$ were indicated for each comparison

outcome was revealed (dead mice: $3.79 \pm 0.23 \log _{10}$ copies $/ \mu \mathrm{L}$; survival mice: $3.39 \pm 0.21 \log _{10}$ copies $/ \mu \mathrm{L} ; P=0.002$ ). Taken together, these results demonstrated that treatment with these CCBs reduced SFTSV viral load and concomitantly reduced fatality rate in the humanized mouse model, a lethal infection animal model for SFTSV.

Nifedipine exhibits clinical antiviral effect in SFTS patients The anti-SFTSV activity in vitro and in mouse model strongly suggested that CCBs could serve as prominent candidates for treating SFTS clinically. We therefore carried out a retrospective clinical investigation on three groups of strictly defined patients to evaluate the effect of nifedipine in treating SFTS. By thoroughly reviewing the medical records of 2087 adult patients who had been admitted into the largest sentinel hospital for SFTS from April 2011 to October 2017, 83 nifedipine-treated, 48 nonnifedipine-treated, and 249 general SFTS patients as defined in detail in Materials and Methods, were included for investigation and these patients did not receive treatment with other CCBs or related drugs (Fig. 5a). For nifedipine-treated, general SFTS, and non-nifedipine groups, the mean \pm SD age was $64.5 \pm 8.7,64.9 \pm$ 10.4 , and $62.6 \pm 8.9$ years, the female proportion was $68.7 \%$, $68.7 \%$, and $72.9 \%$, and the median (interquartile-range) delay from symptom onset to hospital admission was 5 (4-6), 5 (4-6), and 5 (4-7) days, respectively. None of the three variables showed significant inter-group difference (each $P>0.05$, Supplementary information, Table S2). The frequencies of clinical manifestations that were recorded before or at admission, including non-specific features (fever, feeble, myalgias, etc.), lymphadenopathy, respiratory syndrome, gastrointestinal symptoms, hemorrhagic signs, and neurological symptoms, were comparable between nifedipinetreated group and general SFTS group or non-nifedipine group (all $P>0.05$, Supplementary information, Table S2). Almost all laboratory parameters tested at admission were comparable, except that the WBC and RBC (red blood cell) counts and the serum calcium level in nifedipine-treated group were higher than those in general SFTS group $(P=0.011, P=0.028$, and $P=0.006$, respectively, Supplementary information, Table S3). The mean viral load ( $\log _{10}$-transformed) tested at admission and the proportion of the patients with different viremia levels in nifedipine-treated group were comparable between nifedipine-treated group and general SFTS group, as well as between nifedipine-treated group and non-nifedipine group (all $P>0.05$, Supplementary information, Table S4). The commonly prescribed therapies during hospitalization included recombinant human granulocyte colony-stimulating factor, XueBiJing (a traditional Chinese medicine for treating sepsis), immunoglobulin, ribavirin, corticosteroid, thrombopoietin, and transfusion of PLTs or plasma. All of the therapies were observed with comparable frequencies between nifedipine-treated group and general SFTS group, as well as between nifedipine-treated group and non-nifedipine group (all $P$ $>0.05$, Supplementary information, Table S5). These comparable baseline features eliminated bias on the efficacy analysis.

When SFTS-related fatality was used as the primary outcome, a beneficial effect in reducing the CFR was determined from nifedipine therapy; the CFR was remarkably decreased by $>5$-fold in nifedipine-treated group $(3 / 83,3.6 \%)$ than that in general SFTS group $(49 / 249,19.7 \%, P<0.001)$ or non-nifedipine group $(10 / 48$, $20.8 \%, P<0.001)$. Notably, the significant decrease of CFR was also observed in the nifedipine-treated patients $(1 / 41,2.4 \%)$ with high 
a

2087 SFTS adult patients admitted to 154 hospital during 2011-2017 were reviewed

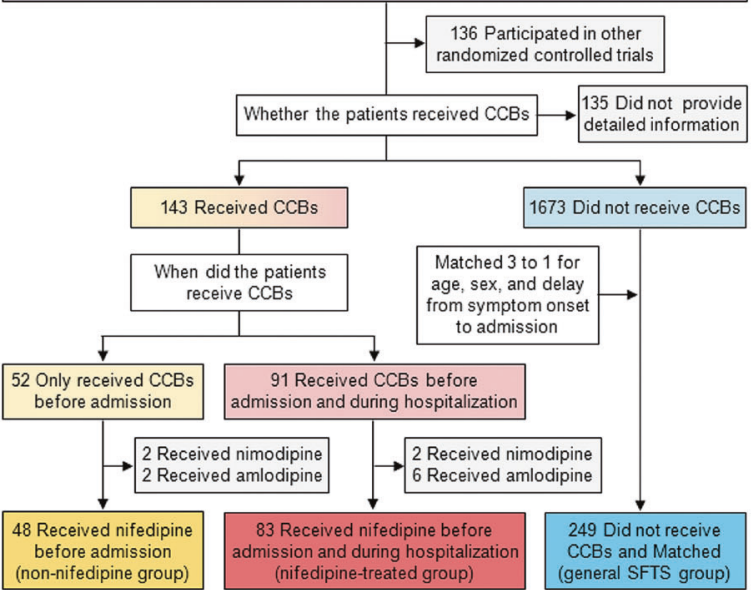

c

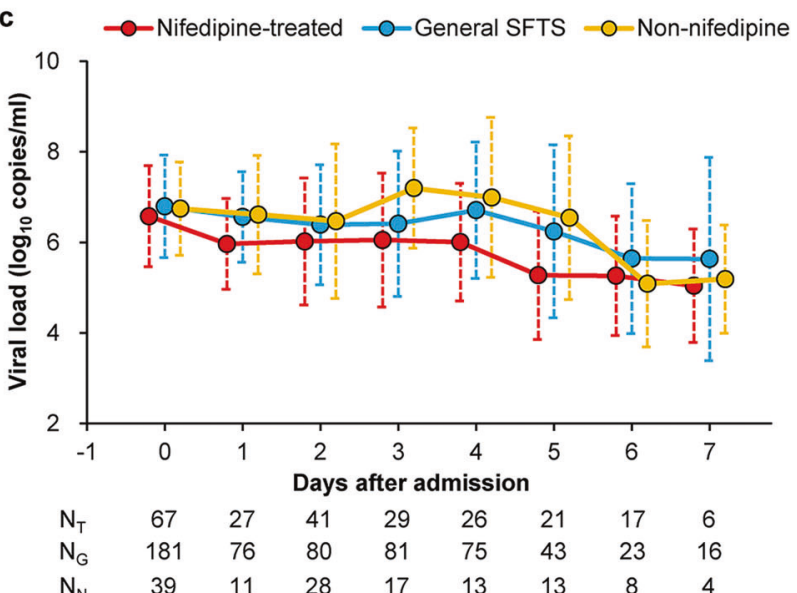

b
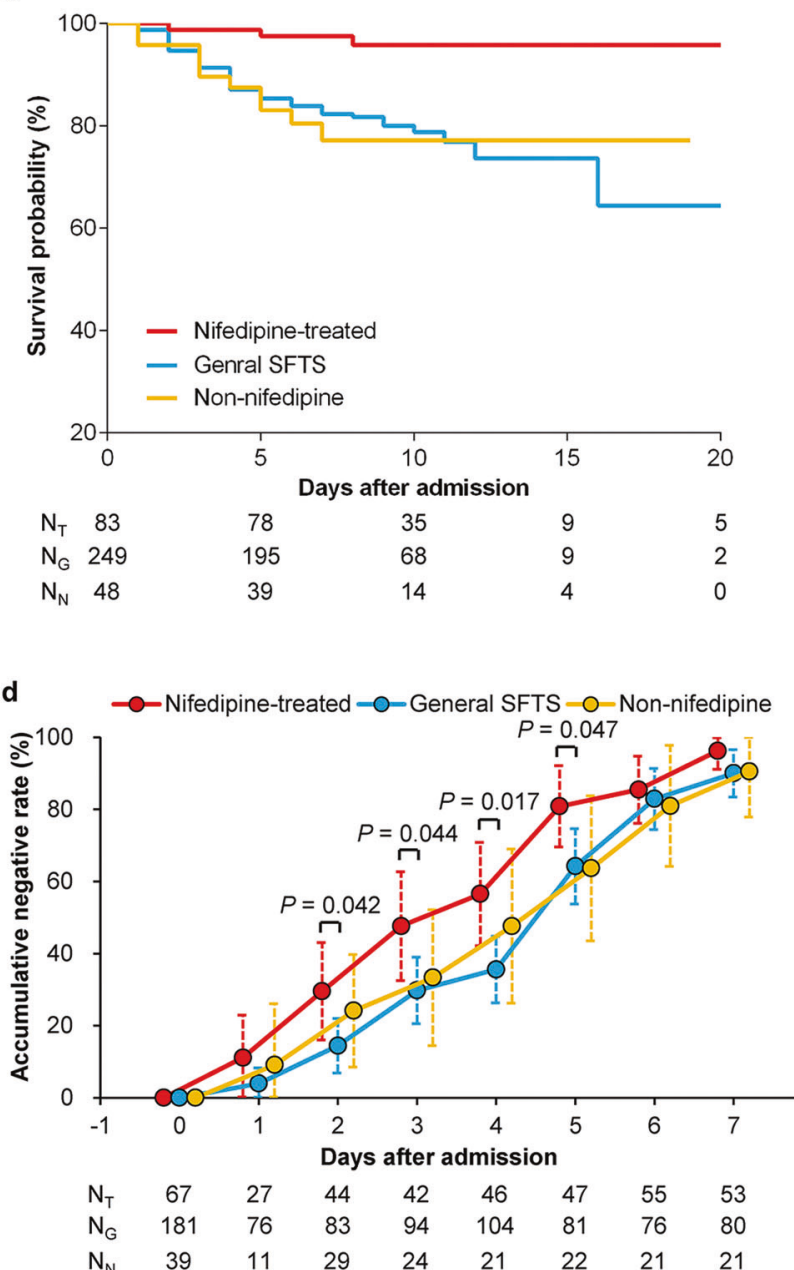

Fig. 5 Clinical effect analysis of nifedipine treatment on SFTS-related fatality and clearance of SFTSV. a Flowcharts of recruitment and grouping of SFTS patients. A total of 2087 patients who had been admitted into the sentinel hospital for SFTS, were included in the retrospective clinical investigation that was performed to evaluate the clinical effect of nifedipine in treating SFTS. By thoroughly reviewing the medical records of these patients, 83 nifedipine-treated, 48 non-nifedipine-treated, and 249 general SFTS patients, were included for comparison. CCBs, calcium channel blocks. b Analysis of nifedipine treatment on survival probability. The Kaplan-Meier method was used to analyze time-to-event data. $\mathrm{N}_{T}$, the number of nifedipine-treated patients; $\mathrm{N}_{\mathrm{G}}$, the number of general SFTS patients; $\mathrm{N}_{\mathrm{N}}$, the number of nonnifedipine-treated patients. c Analysis of nifedipine treatment effect on the viral loads in SFTS patients. The GEE model was used to analyze sequentially tested viral loads during hospitalization; statistical analysis results were presented in Supplementary information, Table S7. The circle indicates mean value and the dashed line indicates standard deviation. $\mathbf{d}$ Analysis of nifedipine treatment effect on the viral clearance in SFTS patients. $\chi^{2}$ test was used to compare the frequency difference between two groups, and $P$ values $<0.05$ were shown. The circle indicates frequency and the dashed line indicates $95 \% \mathrm{Cl}$. $\mathrm{N}_{\mathrm{T}}$ indicates number of patients in nifedipine-treated group, $\mathrm{N}_{\mathrm{G}}$ indicates number of patients in general SFTS group, and $\mathrm{N}_{\mathrm{N}}$ indicates number of patients in non-nifedipine group

viremia level $\left(\geq 10^{6}\right.$ copies $\left./ \mathrm{mL}\right)$, when compared to general SFTS patients $(38 / 131,29.0 \%, P<0.001)$ and non-nifedipine-treated patients $(10 / 29,34.5 \%, P<0.001$, Supplementary information, Table S6). Kaplan-Meier analysis similarly demonstrated reduced risk of death in nifedipine-treated group, in comparison with general SFTS group (hazard ratio (HR) 0.148 , 95\% confidence interval $(\mathrm{Cl}) 0.046-0.477, P=0.001)$ or non-nifedipine group (HR $0.148,95 \% \mathrm{Cl} 0.040-0.539, P=0.004$; Fig. 5b).

Given that viral load was strongly associated with survival, ${ }^{6,18,38}$ we performed effect evaluation using serial viral loads as the secondary outcome. The generalized estimating equation (GEE) model based on sequentially tested viral loads during hospitalization revealed that nifedipine treatment significantly reduced the viral loads, in comparison with general SFTS group $(P<0.001)$ or non-nifedipine group $(P=0.002$, Fig. $5 c$, Supplementary information, Table S7). To corroborate this finding, the virus clearance rate was found to be increased following nifedipine treatment; $29.5 \%$,
$47.6 \%, 56.5 \%$, and $80.9 \%$ of the patients turned negative for SFTSV at days 2-5 after therapy, respectively, and all were significantly higher than those in general SFTS group $(14.5 \%, 28.9 \%, 35.6 \%$, and $64.2 \%$, respectively; all $P<0.05$, Fig. 5 d).

Nifedipine treatment significantly improved clinical outcome of patients with severe SFTSV infection

Further, nifedipine treatment was found to decrease the risk of severe complication development. Neurological syndromes, especially coma and lethargy, which were the prominent complications that were associated with fatal outcome, ${ }^{6,18,39}$ were observed with much lower frequencies in nifedipine-treated group, in comparison with those in general SFTS group and non-nifedipine group (1.2\% vs $7.8 \%$ vs $14.9 \%$ and $0 \%$ vs $5.7 \%$ vs $8.5 \%$, respectively, Fig. 6a). Likewise, hematemesis, one of the hemorrhagic manifestations that are related to death, ${ }^{6}$ was found to occur less in the nifedipine-treated group, although the difference was 


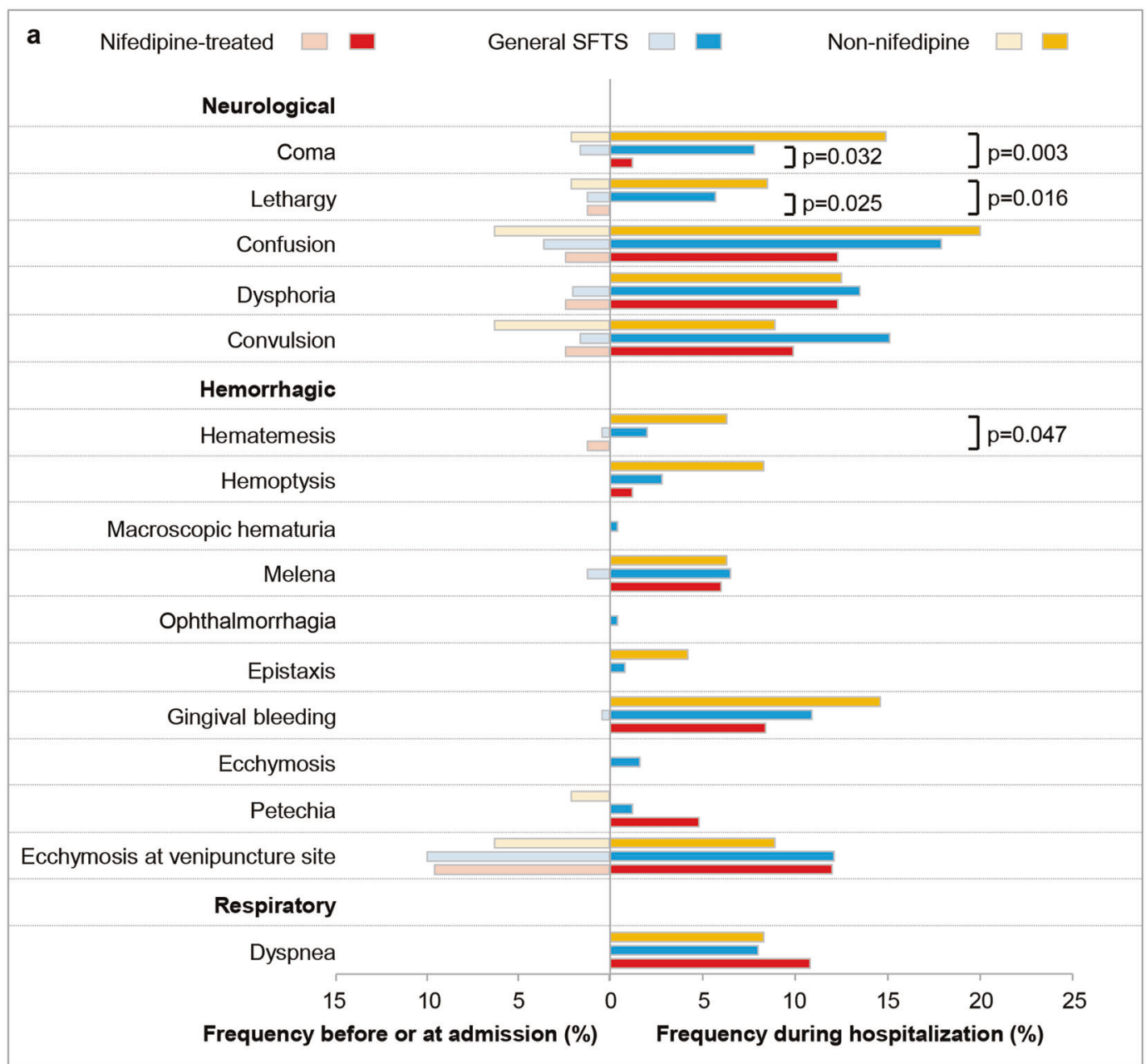

b

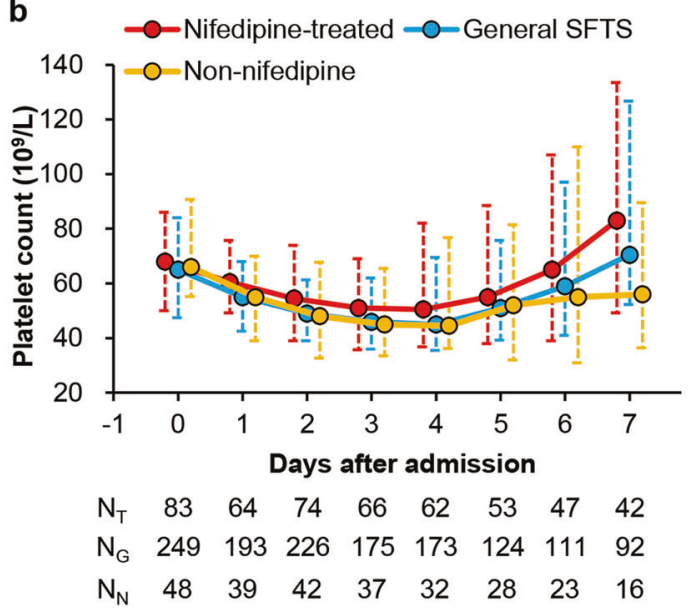

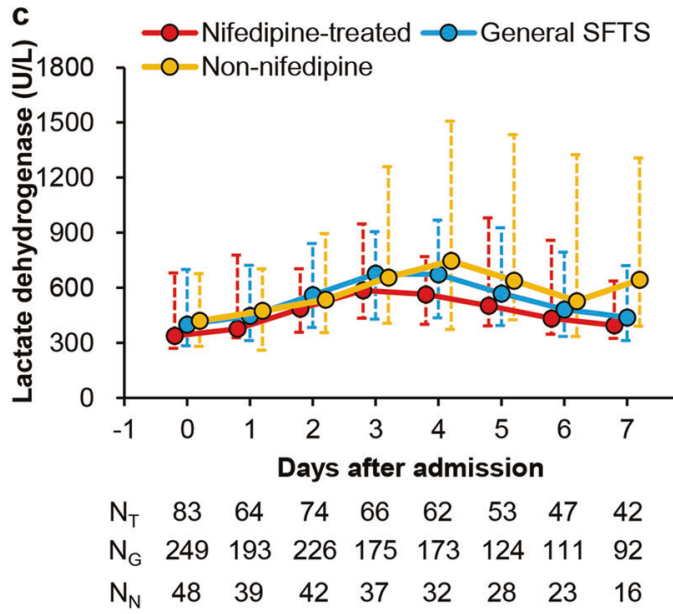

Fig. 6 Clinical effect analysis of nifedipine treatment on severe complication development and laboratory parameter recovery of SFTS patients. a Left: frequencies of severe complications presented before or at admission; right: frequencies of severe complications developed during hospitalization. Comparisons of severe complications that developed during hospitalization in nefidipine-treated group with those in general SFTS group or in non-nifedipine group were performed by $\chi^{2}$ test or Fisher's exact test. $P$ values $<0.05$ were shown. $\mathbf{b}$, $\mathbf{c}$ Sequential data on PLT count (b) and LDH level (c) were analyzed by GEE models; statistical analysis results were presented in Supplementary information, Table S7. The circle indicates median value and the dashed line indicates interquartile-range. $\mathrm{N}_{\mathrm{T}}$ indicates number of patients in nifedipine-treated group, $\mathrm{N}_{\mathrm{G}}$ indicates number of patients in general SFTS group, and $\mathrm{N}_{\mathrm{N}}$ indicates number of patients in nonnifedipine group 
only significant when compared to non-nifedipine group ( $P=$ 0.047, Fig. 6a). The GEE model using sequential laboratory parameters showed that nifedipine-treated group accelerated the recovery of PLT count to normal level compared to general SFTS group $(P=0.045$, Fig. 6b; Supplementary information, Table S7) and the recovery of lactate dehydrogenase (LDH) level to normal level compared to non-nifedipine group $(P=0.015$, Fig. 6c; Supplementary information, Table S7), without revealing significant difference for the other 10 laboratory parameters (Supplementary information, Fig. S12 and Table S7).

It is also noteworthy that the low serum calcium level at admission was associated with an increased risk of death (odds ratio (OR) 4.331, 95\% Cl 2.242-8.369, $P<0.001$ ), based on univariate logistic regression analysis of the 2087 patients. Meanwhile, nifedipine treatment was persistently associated with higher serum calcium levels by comparison between nifedipinetreated group and general SFTS group using GEE model $(P=$ 0.001 , Supplementary information, Fig. S13, Table S7), suggesting that nifedipine treatment may lead to reduced SFTSV infection in a $\mathrm{Ca}^{2+}$ uptake-dependent manner, as observed in vitro.

\section{DISCUSSION}

The CCBs represent a group of widely used anti-hypertensive and anti-atherosclerotic agents. Here, we identified benidipine hydrochloride and nifedipine, two CCBs that are commonly used in clinics, as anti-SFTSV compounds in vitro and showed that treatment with these two CCBs inhibited viral infection in C57BL/6 mice, a non-lethal SFTSV infection mouse model. ${ }^{37}$ To corroborate the in vivo analysis of anti-SFTSV effect, we established SFTSV infection in the humanized mouse model. SFTSV infection of humanized mice caused abnormalities similar to clinical SFTSV patients including reduced PLT and WBC counts and elevated hepatic enzyme levels. ${ }^{2,6,18}$ Furthermore, SFTSV infection resulted in lethal outcome in this model, making it possible for evaluating the correlation between drug effect and fatality rate. Treatment with these two CCBs inhibited SFTSV replication and concomitantly reduced the SFTSV-associated fatality rate in the humanized mouse model. Together, these results clearly demonstrated the anti-SFTSV effects of benidipine hydrochloride and nifedipine in vivo.

Most importantly, we have provided the first clinical evidence indicating that CCBs could be developed as an effective therapy for SFTSV infection. By performing a retrospective clinical investigation on a cohort of 2087 SFTS patients, we found that nifedipine administration enhanced virus clearance, alleviated severe complications, and remarkably reduced death occurrence of SFTS patients by $>5$-fold. These findings are of critical clinical relevance for treating SFTS patients. Currently, effective therapy against severe SFTSV infection is unavailable. Patients with serum viral load of $>10^{6}$ copies $/ \mathrm{mL}$ often suffer from severe complications with a high fatality of $\sim 27 \% .^{6}$ In our study, the fact that nifedipine treatment significantly reduced the CFR from $29.0 \%$ (general SFTS patients) to $2.4 \%$ (nifedipine-treated patients) for patients with high viremia level $\left(>10^{6}\right.$ copies $/ \mathrm{mL}$ ) suggests that nifedipine treatment may be a highly effective therapy for severe SFTSV infection. Notably, the results showed that the anti-SFTSV effect could be attained by short-term administration of nifedipine, based on the findings that for those patients who had ceased nifedipine treatment after SFTS disease onset, a similarly high CFR was observed as with those who had not used nifedipine before. Therefore, the CCB administration is potentially an effective therapy for treating acute and severe SFTSV infection and for reducing SFTSV-related fatality.

Identification of effective anti-SFTSV drugs is critical for treating potential pandemic SFTS or SFTS-like disease transmission at a global level. Further analysis revealed that these two CCBs can also significantly inhibit the replication of three additional phylogenetically representative SFTSV strains, indicating their potentially broad anti-SFTSV efficacy (Supplementary information, Figs. S5, S6). In recent years, other emerging bunyaviruses have continued to threaten human health. A SFTSV-like virus, named Heartland virus (HRTV), was isolated from patients in the United States and similarly caused severe febrile disease and death in humans. ${ }^{40-42}$ Recently, a new tick-borne virus belonging to the Phenuiviridae, Guertu virus (GTV), was identified in China with the potential of transmission among animals and humans. ${ }^{43}$ Additional experiments showed that benidipine hydrochloride significantly inhibited replication of both HRTV and GTV, while nifedipine significantly inhibited GTV replication and showed a mild inhibition effect on HRTV replication (Supplementary information, Fig. S7). These results indicate that these two CCBs may have a broad antiviral effect against other phenuiviruses. SFTSV itself may pose a threat to global public health. The primary vector of SFTSV, the $H$. longicornis tick, ${ }^{8,9}$ is recently found to spread in eight states of the United States. ${ }^{16,17}$ The wide distribution of the natural reservoir and vector of SFTSV opens the possibility of global transmission of this virus. In case of human infections caused by these viruses, CCBs can serve as potentially therapeutic antiviral drugs, with minimal clinical investigation required due to their approved status.

We found that benidipine hydrochloride inhibited both SFTSV internalization and genome replication, but did not affect virus binding, fusion or budding. The exact mechanism by which benidipine hydrochloride inhibits SFTSV replication awaits further investigation. It is reported that West Nile virus (WNV) infection leads to $\mathrm{Ca}^{2+}$ influx, which is required for efficient viral replication. ${ }^{44}$ The fact that SFTSV infection also induced $\mathrm{Ca}^{2+}$ influx suggested a critical role of increased intracellular $\mathrm{Ca}^{2+}$ level for SFTSV replication, which may explain why CCB treatment significantly inhibited SFTSV infection. We found that a series of CCBs can inhibit SFTSV replication (Fig. 3d), although different inhibition efficacies were observed. While the general feature of $\mathrm{CCBs}$ is to reduce intracellular $\mathrm{Ca}^{2+}$ level, they differ in their target calcium channel subtypes, effective drug concentrations and halflife times and these differences may lead to their different antiSFTSV efficacies. ${ }^{45,46}$ Impairing $\mathrm{Ca}_{\mathrm{v}} 1.2$ calcium channel, a target of both nifedipine and benidipine hydrochloride, inhibited SFTSV infection, further suggesting the beneficial role of increased intracellular $\mathrm{Ca}^{2+}$ level for the viral infection. $\mathrm{Ca}^{2+}$ is a ubiquitous intracellular messenger that regulates multiple cellular pathways. ${ }^{47,48}$ The intracellular $\mathrm{Ca}^{2+}$ may affect virus infection through regulating the function of $\mathrm{Ca}^{2+}$-dependent cellular factors that are required for virus infection. This has been demonstrated for viruses of which the replication mechanisms and relevant virus-host interactions have been extensively studied. For example, annexin II, a calcium-dependent phospholipidbinding protein, interacts with phosphatidylserine in HIV particles or with HIV capsid polyprotein precursor to facilitate virus entry or virion assembly, respectively. ${ }^{49,50}$ The cytosolic $\mathrm{Ca}^{2+}$ concentration regulates the interaction between host protein S100A10 and the polymerase of $\mathrm{HBV}$ to regulate virus transcription. ${ }^{51}$ It can be speculated that the intracellular $\mathrm{Ca}^{2+}$ also regulate the function of cellular dependence factors or viral proteins of SFTSV to facilitate virus internalization and genome replication. Further investigation of SFTSV basic virology of the mechanisms of virus internalization and genome replication and identification of related host factors are required for further in-depth investigation of the $\mathrm{Ca}^{2+}$ regulation of SFTSV replication.

These results also suggest that the CCBs may function as hostoriented therapeutics to inhibit SFTSV infection through regulating the intracellular $\mathrm{Ca}^{2+}$ concentration. The host-oriented therapeutic strategy would find broad application against emerging viral infections. As recognized, to meet the urgent need for safe and effective antiviral therapeutics against highly lethal pathogens, such as Ebola virus, Marburg virus, WNV, Junin virus, 
and Lassa fever virus, strategies targeting viral components often result in drug-resistant variants due to the high mutation rate of RNA viruses. ${ }^{52-55}$ In contrast, strategies that inhibit virus infection through regulating host machinery/microenvironment could significantly reduce the chance of yielding resistant viruses. Indeed, no benidipine hydrochloride- or nifedipine-resistant virus was recovered despite SFTSV was serially cultured in the presence of benidipine hydrochloride or nifedipine treatment for more than 20 rounds of passaging (data not shown).

CCBs have been increasingly reported to have antiviral activity against a number of lethal viruses such as Ebola virus, ${ }^{56}$ Marburg virus $^{56,57}$ Junin virus, ${ }^{58} \mathrm{WNV}^{44}$ and Japanese encephalitis viruses. $^{59}$ It was reported that CCBs inhibited entry of Ebola virus, ${ }^{56}$ and inhibited both fusion and entry of Junin virus. ${ }^{58}$ Notably, although the stage of viral infection inhibited by CCBs was characterized, the molecular mechanisms of how CCBs inhibit these viruses remain enigmatic. This may reflect the complex regulation and functionality of the intracellular $\mathrm{Ca}^{2+}$ ions. $\mathrm{Ca}^{2+}$ is widely involved in multiple cellular pathways regulating diverse processes such as transcription, endocytosis and intracellular membrane fusion, through regulating the functionality of $\mathrm{Ca}^{2+}$-dependent cellular proteins. ${ }^{48,49}$ It can be envisioned that CCBs may inhibit these virus infection with different mechanisms through regulating different virus-dependent cellular pathways that depend on intracellular $\mathrm{Ca}^{2+}$ level. It is also possible that these inhibition mechanisms act in a synergistic way. Indeed, the overall inhibition efficacy of benidipine hydrochloride treatment on SFTSV replication is potentially a synergistic effect of inhibition against both virus internalization and genome replication. At the moment, the definite molecular mechanism of CCB inhibition against SFTSV infection is still unclear. Further investigation of SFTSV replication mechanisms and related calcium-dependent virus-host interactions would be fundamental for in-depth understanding of the CCB inhibition mechanism. These efforts are currently ongoing in our laboratories. Importantly, our study is the first to provide clinical evidence in support of using CCBs to treat viral infection in patients.

This study was subject to the limitation that the clinical results on SFTS patients were retrospective with no randomization and potentially flawed by confounding factors despite stringent control for comparable baseline features of the patients (see Materials and Methods for details). On the other hand, no clinical data is available for evaluating the effect of benidipine hydrochloride in treating SFTS patients as this drug is rarely used clinically in China. Further clinical investigation of the potential therapeutic effect of CCBs against SFTS should also thoroughly evaluate factors including the potential drug side effect, drugdrug interactions, in vivo drug concentration, etc. Nevertheless, our study clearly indicates the potential therapeutic effect of nifedipine in treating SFTS patients and strongly supports the design of future clinical trials to evaluate the safety and efficacy of CCB therapy for SFTS patients, for both benidipine hydrochloride and nifedipine. Moreover, combination treatment with other supportive regimens, including the therapeutic plasma exchange or steroid pulse therapy, which has been suggested to be partially effective, ${ }^{60-62}$ could be considered.

\section{MATERIALS AND METHODS}

Cells and viruses

Vero cell was obtained from American Type Culture Collection, Huh7 cell was obtained from China Center for Type Culture Collection and the BSR-T7 cell was provided by Dr Mingzhou Chen (Wuhan University, China). All cells were maintained in Dulbecco's modified Eagle's medium (DMEM; Gibco Invitrogen) supplemented with $10 \%$ fetal bovine serum (FBS; Gibco Invitrogen), $1 \%$ antibiotic/antimycotic (Gibco Invitrogen), at $37^{\circ} \mathrm{C}$ in a humidified
$5 \% \mathrm{CO}_{2}$ incubator. BSR-T7 cells were selected with $1 \mathrm{mg} / \mathrm{mL} \mathrm{G} 418$ (Invitrogen) added every other passage. SFTSV strain HBMC16_human_2015, ${ }^{63}$ SFTSV strain WCH, GTV strain DXM, ${ }^{43}$ or HRTV ${ }^{40}$ were obtained from China Centre for General Virus Culture Collection. The SFTSV strains HNXY2017-50 and HNXY2017-66 were isolated from the serum of patients in Chinese People's Liberation Army 154 Hospital in Xinyang city of Henan Province. All viruses were propagated in Vero cells and used in this study. All experiments with the virus were performed in a biosafety level 3 (BSL-3) facilities, in accordance with the institutional biosafety operating procedures. The coding sequence of SFTSV-NP protein was cloned into $\mathrm{pET} 28 \mathrm{a}$ vector in-frame with an N-terminal His-tag using the following primers: forward: GCGGAATTCATGTCAGAGTGGTCCAGG; reverse: GCGAAGCTITIACAGG TTCCTGTAAGCAGC. His-NP protein was expressed in E. coli BL21 strain and purified with Ni Sepharose (GE Healthcare, 17-5318-01), and used for polyclonal antibody generation in rabbit.

\section{Screening of FDA-approved drugs}

Drug compounds of FDA-approved drug library were purchased from Selleck (https://www.selleckchem.com/screening/fdaapproved-drug-library.html). Vero cells were seeded in 96-well plates at a density of 40,000 cells per well in a total volume of 100 $\mu \mathrm{L}$ per well and incubated overnight at $37^{\circ} \mathrm{C}$ and $5 \% \mathrm{CO}_{2}$. Cell monolayers were treated with the compounds at a final concentration of $10 \mu \mathrm{M}$ for $1 \mathrm{~h}$, and infected with SFTSV at an $\mathrm{MOI}$ of 1 . At $36 \mathrm{hpi}$, cells were fixed and incubated with rabbit antiNP antibody, followed by anti-rabbit Alexa488 (Invitrogen) and DAPI (Sigma). The plates were imaged using Operetta (PerkinElmer) with a $10 \times$ objective. Nine images were acquired per well in both the DAPI and $488 \mathrm{~nm}$ channels. The percentages of infected and DAPI-positive cells were calculated using automated image analysis software (Harmony 3.5, PerkinElmer). A positive control of anti-SFTSV compound (mycophenolic acid) and vehicle (DMSO) were handled in the same way as for the compound library during drug screening in a blinded fashion, with the compound identities unknown to the experimenters. Z-factor was calculated to assess the robustness of the screening assay, and Z-factor between 0.5 and 1 indicates a valid assay with good separation between controls. Mycophenolic acid is a non-nucleoside, non-competitive, reversible inhibitor of eukaryotic IMP dehydrogenase, and has been reported to inhibit multiple viruses, including bunyaviruses, arenaviruses and flaviviruses. ${ }^{64-66}$

Quantification of SFTSV, GTV and HRTV vRNA by qRT-PCR Cells were harvested and the total cellular mRNA was extracted by using the QIAamp Viral RNA Mini Kit (Qiagen), according to the manufacturer's instructions. For quantitation of vRNA, a standard curve was constructed with a plasmid containing full-length nucleotides of the $S$ gene of the respective virus, and qRT-PCR was performed with the One-step Primer Script RT-PCR Kit (Takara) in a LightCycler 480 (Roche). The primers and probes for SFTSV vRNA quantification have been previously described. ${ }^{9}$ The primer sequences for GTV and HRTV are as follows: GTV-S-Forward CTCAGCCTAACATCATTA; GTV-S-Reverse AGTCAACAACCAAGATAT; HRTV-S-Forward TGTGGCAAGATGTCTAAGA; HRTV-S-Reverse CAACTCTCACTGGTGTCA.

MTT assay

Cells pre-seeded in 96-well plates were treated with the desired concentrations of compound for $24 \mathrm{~h}$, and 3-(4,5-dimethylthiazol2-thiazolyl)-2,5-diphenyl-2H-tetrazolium bromide (MTT, SigmaAldrich) was added at a final concentration of $5 \mathrm{mg} / \mathrm{mL}$. After incubation at $37^{\circ} \mathrm{C}$ for $4 \mathrm{~h}$, the supernatant was removed. Then 50 $\mu \mathrm{L}$ of DMSO was added to each well, and the emitted light at 492 $\mathrm{nm}$ was measured with a Thermo Multiskan enzyme-linked immunosorbent assay reader (Thermo). 
Immunological focus assay

Vero cell monolayers were infected by SFTSV and incubated under a methylcellulose overlay. At $48 \mathrm{hpi}$, Vero cell monolayers were fixed with $4 \%$ formaldehyde in PBS, permeabilized by incubation with $0.5 \%$ Triton X-100 in balanced salt solution. Cells were then stained with rabbit anti-NP antibody and horseradish peroxidase (HRP)-labeled secondary antibody.

\section{SFTSV binding assay}

Vero cells were pretreated with benidipine hydrochloride or vehicle (DMSO) at $37^{\circ} \mathrm{C}$ for $1 \mathrm{~h}$. Then the cells were transferred onto ice, and SFTSV was added at an MOI of 5 for $1 \mathrm{~h}$. After being washed with pre-cooled PBS for three times, the relative level of bound viral particles was quantified via qRT-PCR as above described.

\section{SFTSV entry and internalization assays}

Vero cells were pre-treated with benidipine hydrochloride or vehicle (DMSO) for $60 \mathrm{~min}$. After the pre-incubation period, the cells were cooled on ice for $15 \mathrm{~min}$ and SFTSV at an MOI of 5 was added. After SFTSV were incubated with cells at $4{ }^{\circ} \mathrm{C}$ for $1 \mathrm{~h}$, ammonium chloride was added to prevent fusion, and cells were incubated at $37^{\circ} \mathrm{C}$ for $3 \mathrm{~h}$ to allow entry to occur. Cells were then washed with PBS and treated with trypsin to remove bound virions. The internalized virions were measured with qRT-PCR as previously described. For internalization assay with microscopy, Vero cells were infected with SFTSV with the MOI of 20 in the presence of benidipine hydrochloride $(20 \mu \mathrm{M})$ or vehicle (DMSO) and ammonium chloride for $1 \mathrm{~h}$. Cells were then washed with PBS for three times, briefly treated with trypsin and fixed. Afterwards, cells were labeled with anti-NP rabbit antibody and goat antirabbit fluorescent secondary antibody for microscopy analysis. Cross sections of the middle of the cells were imaged in randomly selected areas and intracellular viral particles from each cell were quantitated.

\section{Syncytium formation assay}

Vero cells were infected with SFTSV at an MOI of 5 . At $48 \mathrm{hpi}$, the cells were rinsed once with PBS and treated with benidipine hydrochloride or vehicle (DMSO) for $3 \mathrm{~h}$, followed by the addition of citrate-phosphate buffer $(0.1 \mathrm{M}$ citric acid, $0.2 \mathrm{M}$ sodium dihydrogen orthophosphate, $\mathrm{pH}$ 5.0). After incubation in acidified medium for $15 \mathrm{~min}$, the cells were washed and placed in DMEM containing $10 \%$ FBS. Then, $5 \mathrm{~h}$ later, the cells were fixed with $4 \%$ paraformaldehyde-PBS, and syncytium formation was visualized by light microcopy.

\section{Mini-genome assay}

For MG assay, BSR-T7 cells were seeded in 12-well plates $20 \mathrm{~h}$ prior to transfection. BSR-T7 cells were transfected with T7 MG-eGFP plasmid together with pCAGGs SFTSV-NP and pCAGGs SFTSV-L plasmids, by using Lipofectamine 3000 (Invitrogen). Forty-eight hours post transfection, medium was replaced with medium containing the indicated compounds at the indicated concentrations. At $12 \mathrm{~h}$ post transfection, the relative intensity of eGFP (MG activity) was measured. Images were analyzed with CellProfiler (http://cellprofiler.org/) to quantify eGFP-positive cells and the eGFP signal intensity at the single-cell level. Single-cell signal intensities were then plotted and analyzed.

Measurement of intracellular cytosolic calcium concentration Fluo-4 Direct ${ }^{\mathrm{TM}}$ (Fluo-4 Direct ${ }^{\mathrm{TM}}$ Calcium Assay Kit, Thermo), a calcium-sensitive dye, was used to measure changes in the intracellular cytosolic $\mathrm{Ca}^{2+}$ concentration. Vero cells pre-seeded in $96-$ well plate $(10,000$ cells per well) were washed with Hanks' balanced salt solution (HBSS) and loaded with $100 \mu \mathrm{L}$ of Fluo-4 Direct $^{\mathrm{TM}}$ dye mix according to the manufacturer's protocol. The cells were infected with SFTSV at an MOI of 5, or 20, or mock treated, and then treated with benidipine hydrochloride $(1 \mu \mathrm{M})$, or nifedipine $(10 \mu \mathrm{M})$, or vehicle (DMSO). The Fluo-4 Direct ${ }^{\mathrm{TM}}$ dye in the cells was measured with instrument settings for excitation at $494 \mathrm{~nm}$ and emission at $516 \mathrm{~nm}$. Recordings were collected from each well at $5 \mathrm{~min}$ intervals.

\section{siRNA transfection}

Huh7 cells pre-seeded in 24 -well plates $(100,000$ cells per well) were transfected with siRNAs using Lipofectamine RNAiMAX (Invitrogen) according to the manufacturer's instructions. In the parallel experiment, scramble siRNA was included as a control. Forty-eight hours post transfection, cells were infected with SFTSV or mock treated, and collected and subjected to further analyses at $48 \mathrm{hpi}$. All siRNA oligonucleotides used in the study were designed and synthesized by GenePharma (Suzhou, China). The siRNA sequences are as follows: CACNA1C-homo-320CCACCAAG GUUCCAACUAUTT; CACNA1C-homo-3144GCAGACUAUGUCUUCA CUATT; CACNA1C-homo-4817CCUGAUCAUCAACCUCUUUTT

\section{Western blot}

For western blot analysis, protein samples were subjected to SDSPAGE and then transferred onto a polyvinylidene difluoride (PVDF) membrane (Millipore). After blocking with 5\% BSA in Tris-buffered saline-Tween 20 (TBS-T), the membrane was probed with primary antibodies and then HRP-conjugated secondary antibodies. Protein bands were detected by an enhanced chemiluminescence (ECL) kit (Thermo). Mouse monoclonal antibody to $\mathrm{Ca}_{\mathrm{v}} 1.2$ (CACNA1C) was purchased from Abcam (ab84814), and rabbit polyclonal antibody to a tubulin was purchased from Proteintech (11224-1-AP).

Infection and treatment of C57BL/6 mouse

Five-week-old female C57BL/6 mice (weight median of $16.1 \mathrm{~g}$, range of 15.1-17.3 g) were kept in an environmentally controlled specific-pathogen-free (SPF) animal facility in the Laboratory Animal Center of Academy of Military Medical Sciences (Beijing, China). According to the body weight before the inoculation of virus, mice were divided into five groups (20 mice per group): SFTSV + vehicle $(0.3 \%$ carboxymethyl cellulose solution) group, SFTSV + benidipine hydrochloride group, SFTSV + nifedipine group, DMEM + benidipine hydrochloride group, and DMEM + nifedipine group. In infection experiments, mice were intraperitoneally inoculated with $200 \mu \mathrm{L}$ of virus solution $\left(10^{5} \mathrm{TCID}_{50}\right)$, or the same volume of DMEM. Benidipine hydrochloride and nifedipine were dissolved in $0.3 \%$ carboxymethyl cellulose solution and given by using a stomach probe, with the tolerable dose of $15 \mathrm{mg} / \mathrm{kg} / \mathrm{d}$ and $100 \mathrm{mg} / \mathrm{kg} / \mathrm{d}$, respectively. Treatments were administered twice a day and continued for 7 days. Animal experiment was performed in accordance with the National Institutes of Health guidelines under protocols approved by the Institutional Animal Care and Use Committee.

Daily measuring and sampling

The C57BL/6 mice were monitored daily for signs of disease (hunched posture, ruffled fur, decreased activity, and response to stimuli), body weight, and rectal body temperature after infection. The data of weight were reported as the mean weights of the animals relative to their starting weights. Five mice from each group were sacrificed on $1,3,5$, and $7 \mathrm{dpi}$, respectively. Prior to sacrifice, blood samples (about $50 \mu \mathrm{L}$ per animal) were collected via cardiac puncture from anesthetized mice in EDTA tubes for hematological analysis, and the remaining blood samples were collected for serum separation for biochemical and viremia analysis. Spleen samples were subsequently collected for viral load analysis, histopathology and immunohistochemisty. 
Quantitation of viral load

Twenty microliters of serum sample and $10 \mathrm{mg}$ of spleen sample from each C57BL/6 mouse were used for RNA extraction, and the subsequent quantitation of SFTSV RNA was performed by qRT-PCR as above described. For spleen samples, viral RNA copies were normalized to RNA input (per mg). For serum samples, viral RNA copies were represented as copies per microliter of serum.

Hematology and serum biochemistry

Approximately $50 \mu \mathrm{L}$ of EDTA blood sample collected from each C57BL/6 mouse was analyzed for hemoglobin and counts of PLT, WBC, lymphocyte (LYM), neutrophil (NEU), and erythrocyte at all sampling times by using a Sysmex Xs-800i Analyzer. Approximately $100 \mu \mathrm{L}$ of serum sample collected from each mouse was analyzed for ALT, AST, LDH, creatine kinase (CK), blood urea nitrogen (BUN), and calcium by using a Hitachi 700-210 Analyzer. All results were expressed as mean values.

Histopathology and immunohistochemistry

Spleen tissues collected from the mice were fixed with $4 \%$ formaldehyde in PBS and embedded in paraffin. Sections were stained with $\mathrm{H} \& \mathrm{E}$ or processed for immunohistochemistry (IHC). A rabbit polyclonal antibody against SFTSV NP was used as primary antibody for IHC.

Animal experiment in a humanized mouse model NOD.Cg-PrkdcemIIDMO $/ I 2 r^{\text {em2IDMO }}$ mice (NOD-Prkdc null $I L 2 R \gamma^{\text {null, }}$ $\left.\mathrm{NPI}^{\oplus}\right)^{67}$ used for the construction of humanized mouse were obtained from BEIJING IDMO Co., Ltd and kept in a SPF facility. For the generation of humanized immune system, the animals were subjected to a single intraperitoneal injection with Busulfan (Sigma Aldrich) at $20 \mathrm{mg} / \mathrm{kg}$ body weight at $48 \mathrm{~h}$ before transplantation. Human CD34+ cells isolated from umbilical cord blood (Beijing Novay biotech) with purity over $90 \%$ were transplanted into the mice by tail intravenous injection. Each humanized mouse was examined for the presence of human CD45 + cells and murine CD45+ cells in the mouse peripheral blood by flow cytometry at 4, 8, 12, and 16 weeks post engraftment. The median percentage of human CD45+ cells of humanized mice (weight median of $23.0 \mathrm{~g}$, range of $20.3-25.0 \mathrm{~g}$ ) used in this study was $66.45 \%$ (range of $50.59 \%-83.59 \%$ ).

The humanized mice were divided into three groups: SFTSV + vehicle group $(n=7)$, SFTSV + benidipine hydrochloride group ( $n$ $=6)$, and SFTSV + nifedipine group $(n=6)$, according to the presence of human $\mathrm{CD} 45+$ cells. Virus infection and drug treatment were performed as above mentioned in C57BL/6 mice, and the treatments lasted 10 days. About $50 \mu \mathrm{L}$ caudal vein blood samples were collected for serum separation on $1,3,5,7,9,13$, and $17 \mathrm{dpi}$, respectively, and viral load in serum was quantified as above mentioned.

\section{Patients}

To investigate the clinical effect of nifedipine treatment on SFTS, we conducted a retrospective clinical investigation on the patients who sought treatment for SFTS in the Chinese People's Liberation Army 154 Hospital in Xinyang city of Henan Province, the largest sentinel hospital for SFTS in China. ${ }^{6,18}$ Adult patients with laboratorydiagnosed SFTS according to the guidelines released by Chinese Ministry of Health, 68 were examined for medical record of CCB treatment by a trained research medical staff. SFTS patients who received nifedipine before admission to the hospital and were still using nifedipine during hospitalization were defined as nifedipinetreated group. Two groups of patients were used for comparison. The first group consisted of SFTS patients who previously received nifedipine but had ceased the treatment after admission (nonnifedipine group). SFTS patients free of CCB treatment before admission and during hospitalization, were randomly selected by propensity score matching ( 3 to 1 for age, sex, and delay from symptom onset to admission) with the nifedipine-treated patients, and defined as the second group (general SFTS group). Patients who did not provide accurate information on CCB treatment, or received other CCBs rather than nifedipine, were excluded from the analysis. Patients who were recruited in other randomized controlled trials were also excluded. The research protocol was approved by the human ethics committee of the hospital in accordance with the medical research regulations of China, and all participants provided written informed consent to have their samples and information collected.

\section{Data collection}

Data of demography, clinical manifestations, laboratory testing results, medication use and outcome were retrospectively collected by reviewing medical records and inputted into standardized database. For the patients who discontinued therapy or had been discharged from hospital because of adverse clinical progression, we made follow-up visits within two weeks of discharge by phone call or home visit to determine their final outcome (death or survival). The patients had their serum samples collected at the acute infection before any therapy was administered, and serially collected throughout the hospitalization. These samples were used for the quantitation of viremia by qRT-PCR as above described.

\section{Outcome}

The primary outcome was SFTS-related fatality. Secondary outcome was the changes of viral loads. Other outcomes included clinical severe complications (i.e., dyspnea, hemorrhagic signs, and neurologic symptoms) developed during hospitalization and 12 important laboratory parameters (PLT, WBC, LYM percentage, NEU percentage, AST, ALT, LDH, CK, BUN, creatinine (CREA), albumin (ALB), and alkaline phosphatase (ALP)) that had been identified to be risk factors for death. $6,18,38,39,69,70$

Statistics

Continuous variables were summarized as means and standard deviations or as medians and interquartile-range. Student's $t$-test or nonparametric test (Mann-Whitney test) was used for comparisons of continuous variables between two groups, and ANOVA test or nonparametric test was used for comparisons of continuous variables among multiple groups. Categorical variables were summarized as frequencies and proportions, and were analyzed by $x^{2}$ test or Fisher's exact test. $R^{2}$ was estimated for dose dependent analysis by using nonlinear regression model. We used the Kaplan-Meier method and the log-rank test to analyze timeto-event data for treatment effect analysis. We calculated HRs and $95 \% \mathrm{Cl}$ by using Cox regression models, and calculated ORs and $95 \% \mathrm{Cl}$ by using logistic regression models. Sequential data on daily viral loads and laboratory parameters were analyzed over time with the GEE models. A 2-sided $P$ value $<0.05$ was considered statistically significant. All statistical analyses were performed using SPSS software, version 19.0.

\section{DATA AVAILABILITY}

The data that support the findings of this study are available from the corresponding authors upon request.

\section{ACKNOWLEDGEMENTS}

This work was supported by the Strategic Priority Research Program of the Chinese Academy of Sciences (XDB29010204), the National Natural Science Foundation of China (81825019, 81722041, 31770188, 81472005, 81473023 and 31500144), the National Science and Technology Major Project (2018ZX10101004001005), the National Key R\&D Program of China (2016YFC1200400, 2018YFA0507201 and 2016YFC1201905), the Hundred Talents Program of the Chinese Academy of Sciences (K.P.), the Youth Innovation Promotion Association of the Chinese Academy of Sciences (2018367), the Special Major Program of Wuhan Institute of Virology (WIV- 
135-TP1), the State Key Laboratory of Virology Open Projects (2017IOV003), the Foundation for Innovative Research Groups of the National Natural Science Foundation of China (81621005), and the New Star Plan of Science and Technology of Beijing (Z171100001117089).

\section{AUTHOR CONTRIBUTIONS}

K.P. and W.L. conceived and supervised the study. H.L., L.-K.Z., K.P. and W.L. designed the experiments, analyzed the results, and wrote the manuscript. H.L., L.-K.Z., S.-F.L., W.-W.W., Y.-L.Z., Q.-L.X., K.D., Y.-Y.H., X.-T.Z., Y.-J.F., P.-H.Z., J.-Y.B. and K.P. performed the experiments. H.L., S.-F.Z., Z.-B.W., N.C., C.Y. and W.L. recruited the patients. H.L., L.K.Z., S.-F.Z. and Q.-B.L. performed statistical analysis. G.-F.X. and F.D. contributed to the design of the study and data analysis. All authors had access to the study data, and reviewed and approved the final manuscript.

\section{ADDITIONAL INFORMATION}

Supplementary information accompanies this paper at https://doi.org/10.1038/ s41422-019-0214-z.

Competing interests: The authors declare no competing interests.

\section{REFERENCES}

1. Paules, C. I., Marston, H. D., Bloom, M. E. \& Fauci, A. S. Tickborne diseases confronting a growing threat. N. Engl. J. Med. 379, 701-703 (2018).

2. $\mathrm{Yu}, \mathrm{X}$. J. et al. Fever with thrombocytopenia associated with a novel bunyavirus in China. N. Engl. J. Med. 364, 1523-1532 (2011).

3. Liu, Q., He, B., Huang, S. Y., Wei, F. \& Zhu, X. Q. Severe fever with thrombocytopenia syndrome, an emerging tick-borne zoonosis. Lancet Infect. Dis. 14, 763-772 (2014).

4. Kim, K. H. et al. Severe fever with thrombocytopenia syndrome, South Korea, 2012. Emerg. Infect. Dis. 19, 1892-1894 (2013).

5. Takahashi, T. et al. The first identification and retrospective study of severe fever with thrombocytopenia syndrome in Japan. J. Infect. Dis. 209, 816-827 (2014).

6. $\mathrm{Li}, \mathrm{H}$. et al. Epidemiological and clinical features of laboratory-diagnosed severe fever with thrombocytopenia syndrome in China, 2011-17: a prospective observational study. Lancet Infect. Dis. 18, 1127-1137 (2018).

7. 2017 Annual review of diseases prioritized under the Research and Development Blueprint (WHO Meeting report, World Health Organization, 2017).

8. Luo, L. M. et al. Haemaphysalis longicornis ticks as reservoir and vector of severe fever with thrombocytopenia syndrome virus in China. Emerg. Infect. Dis. 21, 1770-1776 (2015).

9. Zhuang, L. et al. Transmission of severe fever with thrombocytopenia syndrome virus by Haemaphysalis longicornis ticks, China. Emerg. Infect. Dis. 24, https://doi. org/10.3201/eid2405.151435 (2018).

10. Park, S. W. et al. Prevalence of severe fever with thrombocytopenia syndrome virus in Haemaphysalis longicornis ticks in South Korea. Ticks Tick. Borne Dis. 5, 975-977 (2014).

11. Tateno, M. et al. Molecular survey of arthropod-borne pathogens in ticks obtained from Japanese wildcats. Ticks Tick. Borne Dis. 6, 281-289 (2015).

12. Hammer, J. F., Emery, D., Bogema, D. R. \& Jenkins, C. Detection of Theileria orientalis genotypes in Haemaphysalis longicornis ticks from southern Australia. Parasit. Vectors 8, 229 (2015).

13. Heath, A. Biology, ecology and distribution of the tick, Haemaphysalis longicornis Neumann (Acari: Ixodidae) in New Zealand. N. Z. Vet. J. 64, 10-20 (2016).

14. Yun, Y. et al. Phylogenetic analysis of severe fever with thrombocytopenia syndrome virus in South Korea and migratory bird routes between China, South Korea, and Japan. Am. J. Trop. Med. Hyg. 93, 468-474 (2015).

15. Li, Z. et al. Ecology of the tick-borne phlebovirus causing severe fever with thrombocytopenia syndrome in an endemic area of China. PLoS Negl. Trop. Dis. 10, e0004574 (2016).

16. Rainey, T., Occi, J. L., Robbins, R. G. \& Egizi, A. Discovery of Haemaphysalis longicornis (Ixodida: Ixodidae) parasitizing a sheep in New Jersey, United States. J. Med. Entomol. 55, 757-759 (2018).

17. National Haemaphysalis longicornis (longhorned tick) Situation Report - August 29, 2018 (United States Department of Agriculture, 2018).

18. Liu, W. et al. Case-fatality ratio and effectiveness of ribavirin therapy among hospitalized patients in china who had severe fever with thrombocytopenia syndrome. Clin. Infect. Dis. 57, 1292-1299 (2013).

19. Tani, H. et al. Efficacy of T-705 (Favipiravir) in the treatment of infections with lethal severe fever with thrombocytopenia syndrome virus. mSphere 1, e00061-15 (2016).
20. Smee, D. F., Jung, K. H., Westover, J. \& Gowen, B. B. 2'-Fluoro-2'-deoxycytidine is a broad-spectrum inhibitor of bunyaviruses in vitro and in phleboviral disease mouse models. Antivir. Res. 160, 48-54 (2018).

21. Richardson, F. C. et al. Quantification of 2'-fluoro-2'-deoxyuridine and 2'-fluoro-2'deoxycytidine in DNA and RNA isolated from rats and woodchucks using LC/MS/ MS. Chem. Res. Toxicol. 15, 922-926 (2002).

22. Baba, M. et al. Establishment of an antiviral assay system and identification of severe fever with thrombocytopenia syndrome virus inhibitors. Antivir. Chem. Chemother. 25, 83-89 (2017).

23. Yuan, S. et al. Screening of an FDA-approved drug library with a two-tier system identifies an entry inhibitor of severe fever with thrombocytopenia syndrome virus. Viruses 11, E385 (2019).

24. Barrows, N. J. et al. A Screen of FDA-approved drugs for inhibitors of Zika virus infection. Cell Host Microbe 20, 259-270 (2016).

25. Johansen, L. M. et al. A screen of approved drugs and molecular probes identifies therapeutics with anti-Ebola virus activity. Sci. Transl. Med 7, 290 ra89 (2015).

26. Zhang, J. H., Chung, T. D. \& Oldenburg, K. R. A simple statistical parameter for use in evaluation and validation of high throughput screening assays. J. Biomol. Screen. 4, 67-73 (1999).

27. Yuan, F. \& Zheng, A. Entry of severe fever with thrombocytopenia syndrome virus. Virol. Sin. 32, 44-50 (2017).

28. Tani, $\mathrm{H}$. et al. Characterization of glycoprotein-mediated entry of severe fever with thrombocytopenia syndrome virus. J. Virol. 90, 5292-5301 (2016).

29. Liu, J. et al. Single-particle tracking reveals the sequential entry process of the Bunyavirus severe fever with thrombocytopenia syndrome virus. Small 15, e1803788 (2019).

30. Hermann, L. L. \& Coombs, K. M. Inhibition of reovirus by mycophenolic acid is associated with the M1 genome segment. J. Virol. 78, 6171-6179 (2004).

31. Yao, K., Nagashima, K. \& Miki, H. Pharmacological, pharmacokinetic, and clinical properties of benidipine hydrochloride, a novel, long-acting calcium channel blocker. J. Pharm. Sci. 100, 243-261 (2006).

32. Zhao, W. \& Zhen, J.-C. Analysis of the utilization of oral antihypertensive drugs in more than 40 hospitals from Beijing area during 2008-2011. China Pharm. 24, 506-509 (2013).

33. Li, X.-P., Yu, Z.-Q. \& Liu, D. Analysis of the application of antihypertensive drugs in 34 hospitals of Wuhan city during 2011 to 2013. China Pharm. 20, 2748-2751 (2015).

34. Seino, H. et al. Effect of benidipine hydrochloride, a long-acting T-type calcium channel blocker, on blood pressure and renal function in hypertensive patients with diabetes mellitus. Anal. Switch. cilnidipine benidipine. Arzneim. 57, 526-531 (2007).

35. Hayashi, K. et al. $\mathrm{Ca}^{2+}$ channel subtypes and pharmacology in the kidney. Circ. Res. 100, 342-353 (2007).

36. Helton, T. D., Xu, W. \& Lipscombe, D. Neuronal L-type calcium channels open quickly and are inhibited slowly. J. Neurosci. 25, 10247-10251 (2005).

37. Jin, C. et al. Pathogenesis of emerging severe fever with thrombocytopenia syndrome virus in C57/BL6 mouse model. Proc. Natl Acad. Sci. USA 109, 10053-10058 (2012).

38. Zhang, Y. Z. et al. Hemorrhagic fever caused by a novel Bunyavirus in China: pathogenesis and correlates of fatal outcome. Clin. Infect. Dis. 54, 527-533 (2012).

39. Gai, Z. T. et al. Clinical progress and risk factors for death in severe fever with thrombocytopenia syndrome patients. J. Infect. Dis. 206, 1095-1102 (2012).

40. McMullan, L. K. et al. A new phlebovirus associated with severe febrile illness in Missouri. N. Engl. J. Med. 367, 834-841 (2012).

41. Muehlenbachs, A. et al. Heartland virus-associated death in tennessee. Clin. Infect. Dis. 59, 845-850 (2014).

42. Fill, M. A. et al. Novel clinical and pathologic findings in a Heartland virusassociated death. Clin. Infect. Dis. 64, 510-512 (2017).

43. Shen, S. et al. A novel tick-borne phlebovirus, closely related to severe fever with thrombocytopenia syndrome virus and Heartland virus, is a potential pathogen. Emerg. Microbes Infect. 7, 95 (2018).

44. Scherbik, S. V. \& Brinton, M. A. Virus-induced $\mathrm{Ca}^{2+}$ influx extends survival of west nile virus-infected cells. J. Virol. 84, 8721-8731 (2010).

45. Zamponi, G. W. Targeting voltage-gated calcium channels in neurological and psychiatric diseases. Nat. Rev. Drug Discov. 15, 19-34 (2016).

46. Elliott, W. J. \& Ram, C. V. Calcium channel blockers. J. Clin. Hypertens. 13, 687-689 (2011).

47. Bagur, R. \& Hajnoczky, G. Intracellular $\mathrm{Ca}^{2+}$ sensing: Its role in calcium homeostasis and signaling. Mol. Cell 66, 780-788 (2017).

48. Clapham, D. E. Calcium signaling. Cell 131, 1047-1058 (2007).

49. Zhou, Y., Frey, T. K. \& Yang, J. J. Viral calciomics: interplays between $\mathrm{Ca}^{2+}$ and virus. Cell Calcium 46, 1-17 (2009).

50. Ma, G. et al. phage HIV-1 infection. J. Exp. Med. 200, 1337-1346 (2004). 
51. Choi, J. et al. Association of hepatitis B virus polymerase with promyelocytic leukemia nuclear bodies mediated by the $S 100$ family protein p11. Biochem Biophys. Res. Commun. 305, 1049-1056 (2003).

52. Perez, M., Craven, R. C. \& de la Torre, J. C. The small RING finger protein Z drives arenavirus budding: implications for antiviral strategies. Proc. Natl Acad. Sci. USA 100, 12978-12983 (2003).

53. Lingappa, U. F. et al. Host-rabies virus protein-protein interactions as druggable antiviral targets. Proc. Natl Acad. Sci. USA 110, E861-E868 (2013).

54. Andrei, G. \& De Clercq, E. Molecular approaches for the treatment of hemorrhagic fever virus infections. Antivir. Res. 22, 45-75 (1993).

55. Garcia, M. et al. Productive replication of Ebola virus is regulated by the c-Abl1 tyrosine kinase. Sci. Transl. Med. 4, 123ra24 (2012).

56. Sakurai, Y. et al. Ebola virus. Two-pore channels control Ebola virus host cell entry and are drug targets for disease treatment. Science 347, 995-998 (2015).

57. DeWald, L. E. et al. The calcium channel blocker Bepridil demonstrates efficacy in the murine model of Marburg virus disease. J. Infect. Dis. 218, S588-S591 (2018).

58. Lavanya, M., Cuevas, C. D., Thomas, M., Cherry, S. \& Ross, S. R. siRNA screen for genes that affect Junin virus entry uncovers voltage-gated calcium channels as a therapeutic target. Sci. Transl. Med. 5, 204ra131 (2013).

59. Wang, S. et al. Screening of FDA-approved drugs for inhibitors of Japanese encephalitis virus infection. J. Virol. 91, e01055-17 (2017)

60. Park, S. Y. et al. Use of plasma therapy for severe fever with thrombocytopenia syndrome encephalopathy. Emerg. Infect. Dis. 22, 1306-1308 (2016).
61. Oh, W. S. et al. Effect of early plasma exchange on survival in patients with severe fever with thrombocytopenia syndrome: a multicenter study. Yonsei Med. J. $\mathbf{5 8}$ 867-871 (2017).

62. Nakamura, S. et al. Steroid pulse therapy in patients with encephalopathy associated with severe fever with thrombocytopenia syndrome. J. Infect. Chemother. 24, 389-392 (2018)

63. Zhang, Y. et al. Isolation, characterization, and phylogenic analysis of three new severe fever with thrombocytopenia syndrome bunyavirus strains derived from Hubei Province, China. Virol. Sin. 32, 89-96 (2017).

64. Livonesi, M. C., Moro de Sousa, R. L. \& Moraes Figueiredo, L. T. In vitro study of antiviral activity of mycophenolic acid on Brazilian orthobunyaviruses. Intervirology 50, 204-208 (2007).

65. Sepulveda, C. S. et al. Inhibition of Junin virus RNA synthesis by an antiviral acridone derivative. Antivir. Res. 93, 16-22 (2012).

66. Takhampunya, R., Ubol, S., Houng, H. S., Cameron, C. E. \& Padmanabhan, R. Inhibition of dengue virus replication by mycophenolic acid and ribavirin. J. Gen. Virol. 87, 1947-1952 (2006).

67. Guo, S. et al. Oncological and genetic factors impacting PDX model construction with NSG mice in pancreatic cancer. FASEB J. 33, 873-884 (2019).

68. Ministry of Health PRC. Guideline for prevention and treatment of severe fever with thrombocytopenia syndrome (2010 vesion). Chin. J. Clin. Infect. Dis. 4, 193-194 (2011).

69. Cui, N. et al. Severe fever with thrombocytopenia syndrome bunyavirus-related human encephalitis. J. Infect. 70, 52-59 (2015).

70. Ding, S. et al. Age is a critical risk factor for severe fever with thrombocytopenia syndrome. PLOS ONE 9, e111736 (2014). 